\title{
Matching and running sensitivity in non-renormalizable inflationary models
}

\author{
Jacopo Fumagalli, ${ }^{a}$ Marieke Postma $^{b}$ and Melvin van den Bout ${ }^{b}$ \\ ${ }^{a}$ Institut d'Astrophysique de Paris, GReCO, UMR 7095 du CNRS et de Sorbonne Université, \\ 98bis boulevard Arago, Paris 75014, France \\ ${ }^{b}$ Nikhef, Science Park 105, Amsterdam 1098 XG, The Netherlands \\ E-mail: fumagall@iap.fr, mpostma@nikhef.nl, melvinvandenbout@gmail.com
}

ABSTRACT: Most of the inflationary models that are in agreement with the Planck data rely on the presence of non-renormalizable operators. If the connection to low energy particle physics is made, the renormalization group (RG) introduces a sensitivity to ultraviolet (UV) physics that can be crucial in determining the inflationary predictions. We analyse this effect for the Standard Model (SM) augmented with non-minimal derivative couplings to gravity. Our set-up reduces to the SM for small values of the Higgs field, and allows for inflation in the opposite large field regime. The one-loop beta functions in the inflationary region are calculated using a covariant approach that properly accounts for the non-trivial structure of the field space manifold. We run the SM parameters from the electroweak to the inflationary scale, matching the couplings of the different effective field theories at the boundary between the two regimes, where we also include threshold corrections that parametrize effects from UV physics. We then compute the spectral index and tensor-toscalar ratio and find that RG flow corrections can be determinant: a scenario that is ruled out at tree level can be resurrected and vice versa.

KeYwords: Cosmology of Theories beyond the SM, Higgs Physics, Renormalization Group, Effective Field Theories

ARXIV EPRINT: 2005.05905 


\section{Contents}

1 Introduction 1

2 The model: Standard Model with non-minimal derivative couplings 4

2.1 Standard Model with non-trivial kinetic sector 6

$\begin{array}{lll}2.2 & \text { Tree level analysis and different regimes } & 7\end{array}$

3 Covariant formalism $\quad 7$

3.1 Large field regime and counterterms 8

$\begin{array}{ll}3.2 & \text { Covariant fields }\end{array}$

4 Renormalization group flow $\quad 10$

$\begin{array}{ll}4.1 \text { Renormalization group equations } & 11\end{array}$

$\begin{array}{lll}\text { 4.1.1 Case A } & 12\end{array}$

$\begin{array}{lll}4.1 .2 & \text { Case B } & 13\end{array}$

$\begin{array}{lll}\text { 4.1.3 Case C and D } & 14\end{array}$

$\begin{array}{lll}4.2 & \text { Beta-functions summary } & 14\end{array}$

5 Predictions for inflation $\quad \mathbf{1 5}$

$\begin{array}{lll}5.1 & \text { Renormalization group dependence: analytical estimate } & 15\end{array}$

$\begin{array}{ll}\text { 5.2 Matching and running: numerical results for case A } & 16\end{array}$

$\begin{array}{lll}\text { 5.2.1 Boundary conditions at the electroweak scale } & 18\end{array}$

$\begin{array}{ll}\text { 5.2.2 Threshold corrections } & 19\end{array}$

5.3 Matching and running: other cases 20

6 Conclusions 23

A Calculation of the self-energies $\quad 24$

A.1 Renormalized action 24

$\begin{array}{lll}\text { A.2 } & \text { Feynman rules } & 25\end{array}$

A.3 Coupling strengths 26

A.4 Two-point functions in the Abelian-Higgs model 27

$\begin{array}{ll}\text { A.5 Two-point functions in the SM } & 27\end{array}$

\section{Introduction}

One of the main lessons learned from the Planck constraints on inflation [1] is that quadratic and quartic inflation, arguably the simplest approaches, are ruled out by the data. Most 
of the successful inflationary models, whether single or multi-field, instead rely on nonrenormalizable operators to obtain predictions in agreement with Planck (see [2] for a large list of models).

It is well known that inflation is sensitive to ultraviolet (UV) physics. Corrections from high energy degrees of freedom tend to increase the inflaton mass, thus ruining the inflationary dynamics needed to sustain a long enough period of inflation [3]. There are two aspects to this so-called eta-problem (see [4] for a review). First, integrating out heavy physics above a given cutoff scale shifts the parameters in the low energy effective field theory (EFT) by an amount proportional to the strong coupling scale. That is the famous hierarchy problem in the context of cosmology. Second, even Planck suppressed irrelevant operators can easily spoil the flatness of the inflaton potential and completely change the inflationary dynamics.

In this work we focus on a different kind of UV sensitivity which stems from the nonrenormalizable character of an inflationary model and comes into play when the connection to the low-energy (beyond the) Standard Model (SM) degrees of freedom is made using the renormalization group ( $\mathrm{RG}$ ) flow $[5,6]$. When the $\mathrm{RG}$ improved action is used to incorporate perturbative quantum corrections, the running of the couplings can affect the naive tree level inflationary predictions. To compute meaningful observables one must determine the RG equations in the inflationary regime, and understand the effects of UV physics at the cutoff scale $\mathcal{M}$, set by the non-renormalizable operators, on the running. Although our approach to analyse this effect is generic, for concreteness we will concentrate on the SM non-minimally coupled to gravity via derivative interactions [7-9].

The presence of a non-renormalizable operators allows to distinguish different regimes characterized by small (field-dependent) parameters. To be concrete, consider the toy model Lagrangian

$$
\mathcal{L}=-\frac{1}{2}\left(1+\frac{\phi}{\mathcal{M}}\right)^{2}(\partial \phi)^{2}+V(\phi) .
$$

In the small field regime $\delta \equiv \phi / \mathcal{M} \ll 1$ the higher-dimensional interaction is a small correction, whereas in the large field regime $\delta^{-1} \ll 1$ it gives the dominant contribution to the kinetic term. In both domains the model may be renormalizable in the EFT sense, by which we mean that in each field region ${ }^{1}$ it is possible to define a small parameter in such a way that at every order in that parameter the theory can be renormalized with a finite number of counterterms. In the example above, loop corrections can be organized in a series expansion in $\delta\left(\delta^{-1}\right)$ in the small (large) field regime. This can be seen, for instance, by considering the one-loop contribution to the effective potential

$$
V^{1 \text { loop }}=\frac{\left(V^{\prime \prime}\right)^{2}}{32 \pi^{2} \epsilon}=\frac{2 \lambda V_{\text {tree }}}{\pi^{2} \epsilon} \frac{\left(\delta+\frac{3}{2}\right)^{2}}{(\delta+1)^{6}}=\frac{\lambda V_{\text {tree }}}{\pi^{2} \epsilon} \cdot \begin{cases}\sum_{k=0}^{\infty} c_{k} \delta^{k} & \delta \equiv \phi / \Lambda \ll 1 \\ \sum_{k=0}^{\infty} \bar{c}_{k}\left(\delta^{-1}\right)^{k}, & \delta^{-1} \ll 1,\end{cases}
$$

where we used dimensional regularization with $d=4-\epsilon$, and a prime denotes a derivative with respect to the canonically normalized field. However, crossing the boundary between

\footnotetext{
${ }^{1}$ If there are several non-renormalizable operators there can be more than two regimes. Important is that the small field and the inflationary regime both have renormalizable EFT descriptions. On the boundaries, and in the midfield ranges where the EFT description fails, UV physics becomes relevant.
} 
the two regimes at $\delta \approx 1$ a full tower of higher-order operators becomes relevant and the model is not renormalizable in the EFT sense. It follows that UV physics is important around the boundary and may affect the running of the couplings in this regime appreciably. $^{2}$ This will then modify the value of the parameters in the RG improved inflationary potential, and thus potentially the predictions of the model.

The possibility that inflation can be sensitive to the six-dimensional SM-EFT operators [10] was first noted in [5] and further studied in [6]. The net effect of those operators is to smear the low energy parameters at the scale marking the boundary between the two regimes. This can equivalently be parametrized in a simpler way by a shift of the low energy parameters at the scale $\mathcal{M}$ [11-14] (see also [15] for a discussion in the context of new Higgs inflation), an approach we will follow in this paper.

The RG flow analysis is particularly relevant for inflationary models whose parameters are (or can be) measured in low energy experiments such as the Large Hadron Collider. Prime examples are models where the Higgs boson plays the role of the inflaton $[7,16,17]$ (see [18] for a recent review); due to an additional non-renormalizable coupling between the SM Higgs boson and the gravity sector, the running of the SM parameters is sensitive to UV physics in the mid-field regime [19-26]. The feasibility of connecting low energy physics to inflation was questioned in [27, 28], and it has motivated various analyses of UV corrections to the inflationary predictions [5, 6, 13-15, 29]. An interesting approach is also the alternative Palatini formulation of gravity [30-34] which gives a higher cutoff scale [35] and therefore less sensitivity to UV physics. It is important to note that the connection to low scale observables is not only an issue for models that embed inflation in (extensions of) the SM; for succesful reheating any inflationary model must be coupled in some way to the SM degrees of freedom.

In this work we continue our investigation of the effects of quantum corrections entering through the RG flow on the inflationary observables pursued in $[6,15,29] .{ }^{3}$ In particular, we complete the analysis for the SM model non-minimally derivatively coupled to gravity, which goes under the name of new Higgs inflation [7-9]. In previous work [15] we showed that the tree level cutoff of the theory is always below the typical energy scales involved at every stage in the universe's history, complementing the analysis in [36]. Furthermore, we pointed out the RG sensitivity of these type of models through analytical considerations (revisited here in section 5.1).

We will derive the Renormalization group equations (RGEs) at leading order in the large field regime, and we explicitly show under which conditions the spectral index and tensor-to-scalar ratio $\left(n_{s}, r\right)$ are sensitive to the running of the couplings. Our main results are eq. (4.17) and figures $4 \mathrm{a}, 4 \mathrm{~b}$ and 5 . The punchline is that computing predictions at tree level is often not enough. Boundary conditions at the electroweak scale, the unknown UV completion as well as the explicit form of the RGEs could easily have an impact on the

\footnotetext{
${ }^{2}$ We assume the UV physics does not alter the inflaton potential at tree level, as this would destroy all predictiveness of the model.

${ }^{3}$ Our study in [6] was later implemented (and confirmed where the analyses overlap) by other groups [13, $14,31,32]$. In particular, the authors in $[13,14]$ study the critical regime where inflation takes place near an inflection point, while [31, 32] analyses the loop corrections in the hilltop scenario in the Palatini formulation.
} 
inflationary parameters. This is qualitatively different from plateau-like models of inflation such as Higgs inflation [6] and the larger class of Cosmological Attractors [29, 37], where the inflationary predictions are insensitive to running effects to lowest order in slow-roll parameters.

Roadmap. The RGEs during inflation and the consequences for the observables are derived in a systematic way. We concentrate on the SM with non-minimal derivative couplings to gravity, reviewed in section 2, but our results can be adapted to other set-ups. We include the possibility that next to the Higgs field, also the fermions and/or gauge bosons have non-minimal derivative couplings. Figure 1 illustrates our approach.

To start, we split the field domain in two regimes in section 2.2, and define the two EFTs in these asymptotic field regions ordered by a small parameter in section 3.1. We identify the independent set of couplings at leading order in the EFT expansion, which may be different in the two regimes. To calculate observables, it is not only important to include the effects of UV physics on the running of the couplings in the boundary region, but is also crucial to understand the relevant couplings in the inflationary regime and how they evolve under the RG flow.

The inflationary action in Higgs inflaton models is non-standard with non-trivial kinetic sectors and higher dimensional interactions. For instance, the derivative couplings considered in this paper generate a non-trivial geometry for the field space manifold. To deal with this complication we use a covariant approach $[26,38,39]$ to compute the oneloop corrections. The formalism is set up in section 3.2, while in section 4 we compute the one-loop beta functions.

In section 5 we numerically calculate the quantum corrected inflationary predictions for different boundary conditions at the electroweak scale, and for different threshold corrections parameterizing the effects of UV physics on the running in the mid-field region. Starting at the electroweak scale the SM RGEs are used to run the couplings to the boundary between the small and large field regime. At the boundary we match the SM couplings to the set of independent couplings of the inflationary EFT, where we include possible threshold corrections to capture the effects of UV physics. We then run the couplings to the energy scale of inflation using the RGEs derived. The spectral index $n_{s}$ and tensorto-scalar ratio $r$ are then computed from the RG improved action, which gives our final result.

We conclude and provide an outlook in section 6.

\section{The model: Standard Model with non-minimal derivative couplings}

We consider the Standard Model (SM) and the Einstein-Hilbert action augmented with non-minimally derivative couplings to gravity for the Higgs, gauge and fermion fields. The action is

$$
S=\int \mathrm{d}^{4} x \sqrt{-g}\left[\frac{1}{2} m_{\mathrm{P}}^{2} R+\mathcal{L}_{\mathrm{SM}}+\mathcal{L}_{\mathrm{KI}}\right]
$$




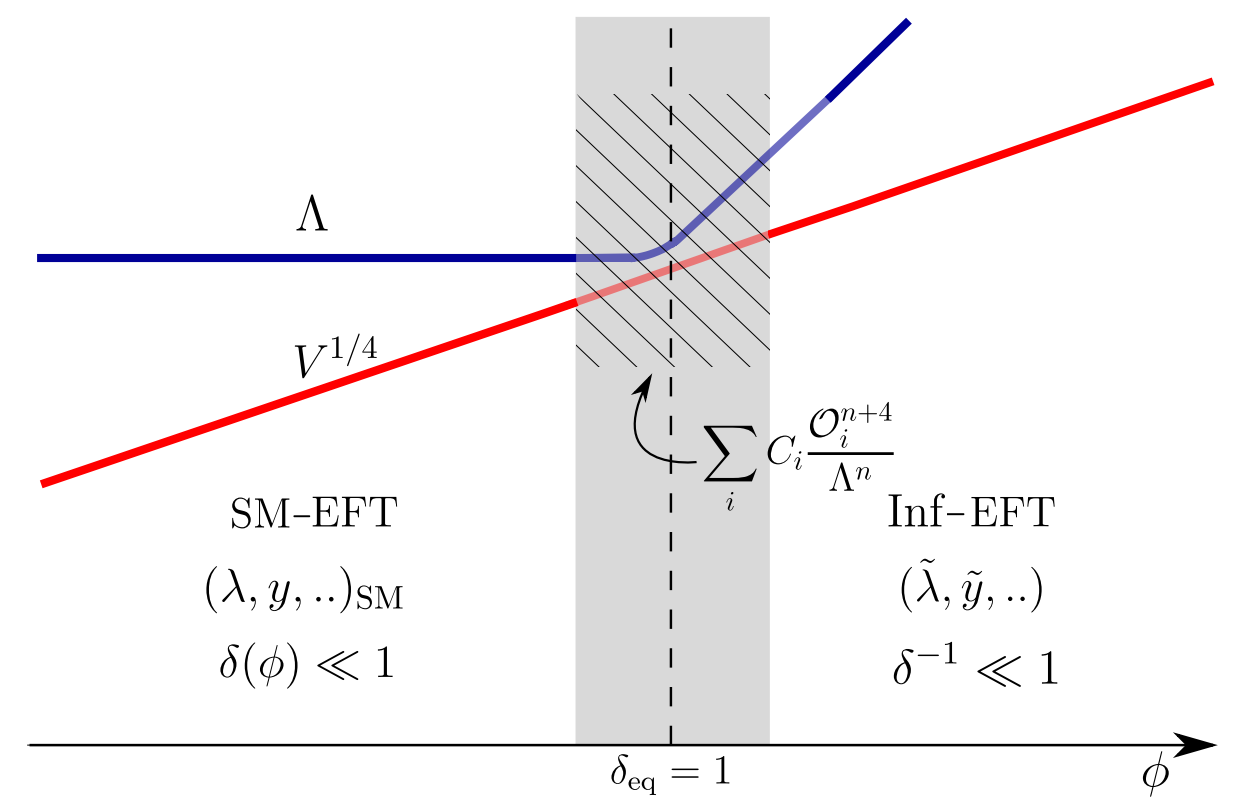

Figure 1. Overview of the renormalization group flow effects on the inflationary predictions. Shown is the typical energy of the potential $V^{1 / 4}$ (red) and the unitarity cutoff (blue) as a function of the parameter $\delta=V / \mathcal{M}^{4}$ that distinguishes between the small field $(\delta \ll 1)$ and large field $(\delta \gg 1)$ region. In each regime an EFT can be constructed which is valid below the cutoff scale, and which depends on a set of independent couplings that are matched at the boundary at $\delta=1$. In the mid field region, indicated by the grey area, the EFT description breaks down, and the effects of UV physics (parameterized by a tower of higher order operators suppressed by the cutoff scale) can be considerable. We implement this by adding threshold corrections to the couplings at the matching scale. We calculalate the inflationary RGEs to subsequently run the couplings from the mid-field to the inflationary scale $\delta_{\star}$, where we calculate $n_{s}$ and $r$ and analyse the effects of the threshold corrections.

with

$$
\mathcal{L}_{\mathrm{KI}}=\frac{G^{\mu \nu}}{M^{2}} D_{\mu} \mathcal{H}^{\dagger} D_{\nu} \mathcal{H}+\sum_{a} \frac{1}{4} \alpha_{A} \frac{3 \mathcal{G}^{\mu \nu \alpha \beta}}{M^{2}} F_{\alpha \beta}^{a} F_{\mu \nu}^{a}+\sum_{i} \alpha_{\psi} \frac{G^{\alpha \beta}}{M^{2}} \psi_{i} i \gamma_{\alpha} D_{\beta} \psi_{i}
$$

where $\mathcal{H}$ is the Higgs doublet, and $R, G^{\mu \nu}, \mathcal{G}^{\mu \nu \alpha \beta}$ the Ricci scalar, the Einstein tensor, and the double-dual Riemann tensor respectively. The summation in the gauge and fermion terms runs over the SM gauge groups and fermions respectively. For simplicity we take the non-minimal couplings for the gauge (fermion) fields universal, i.e. the same for all gauge groups (fermions), the results can easily be generalized. With just a constant value of the non-minimal Higgs coupling this is the original version of the new Higgs inflation proposal of ref. [7], while non-minimal couplings for other sectors were introduced in $[8,9]$. We parameterize the non-minimal gauge boson and fermion couplings as

$$
\alpha_{i}=\alpha_{0 i} \delta^{n_{i} / 2}
$$

for $i=A, F$ and $\delta$ defined as

$$
\delta \equiv \frac{V}{\mathcal{M}^{4}} \simeq \frac{\lambda\left(\mathcal{H}^{\dagger} \mathcal{H}\right)^{2}}{\mathcal{M}^{4}}
$$


where we introduced the scale $\mathcal{M}^{2}=M m_{\mathrm{P}}$, and with $V$ the Standard Model Higgs potential, where we dropped the quadratic term that plays no role at large field values. The couplings vanish for $\alpha_{0 i}=0$ and are constant for $n_{i}=0$, which are arguably the most interesting cases. Thus, in the following we study four cases:

Case A : $\left(\alpha_{A}, \alpha_{f}\right)=(0,0)$,

Case B : $\left(\alpha_{A}, \alpha_{f}\right)=\left(\alpha_{0 A}=\right.$ const., 0$)$,

Case C : $\left(\alpha_{A}, \alpha_{f}\right)=\left(\alpha_{0 A}=\right.$ const., $\alpha_{0 f}=$ const. $)$,

Case D : $\left(\alpha_{A}, \alpha_{f}\right)=\left(\alpha_{0 A} \delta^{n_{A} / 2}, \alpha_{0 f} \delta^{n_{f} / 2}\right), \quad$ with $n_{A} \geq 1 \& n_{f} \geq 0$.

\subsection{Standard Model with non-trivial kinetic sector}

The Higgs-gravity sector can be brought in (approximate) standard form via a disformal transformation of the metric [9]: ${ }^{4}$

$$
g_{\alpha \beta} \longrightarrow g_{\alpha \beta}-\varepsilon_{\alpha \beta} \equiv g_{\alpha \beta}-2 \frac{D_{\alpha} \Phi^{\dagger} D_{\beta} \Phi}{\mathcal{M}^{2}} .
$$

The transformation leads to the action

$$
\begin{aligned}
\mathcal{L}= & -K_{\phi}(\mathcal{H})\left|D_{\mu} \mathcal{H}\right|^{2}-V(\mathcal{H})-\sum_{a} \frac{1}{4} K_{A}(\mathcal{H})\left(F_{\mu \nu}^{a}\right)^{2}+\sum_{i} K_{\psi}(\mathcal{H}) \bar{\psi}_{i}(i \not D) \psi_{i} \\
& -\left(\frac{y_{t}}{\sqrt{2}} \bar{q}_{L} \mathcal{H}_{c} t_{R} L+\text { h.c. }\right) .
\end{aligned}
$$

$F_{\mu \nu}^{a}$ runs over the SM gauge groups, and $\psi_{i}$ over the left- and right-handed fermions. We only added the Yukawa interaction for the top quark, as this gives the dominant contribution to the running, with $q_{L}$ the left-handed doublet, $t_{R}$ the right-handed top quark and $\mathcal{H}_{c}=\left(i \sigma^{2}\right) \mathcal{H}^{*}$. For simplicity from now on $y_{t} \equiv y$. The non-minimal Higgs, gauge and fermion field space metrics are given explicitly by ${ }^{5}$

$$
K_{\phi}=(1+\delta), \quad K_{A}=\left(1+\alpha_{A} \delta\right), \quad K_{\psi}=\left(1+\alpha_{F} \delta\right) .
$$

Eqs. (2.1) and (2.7) are equivalent up to $\mathcal{O}(\varepsilon, \epsilon)$ corrections, with $\varepsilon$ defined in (2.6) and $\epsilon=-\dot{H} / H^{2}$. During inflation $\varepsilon \sim \epsilon \ll 1$ are slow-roll suppressed, however after inflation the corrections can become large [15]. These corrections are degenerate with the quantum corrections in the mid-field regime, and will be aborbed in the threshold corrections that we will introduce in section 5. The SM Higgs doublet can be parameterized

$$
\mathcal{H}=\frac{1}{\sqrt{2}}\left(\begin{array}{c}
\theta_{1}+i \theta_{2} \\
\varphi+i \theta_{3}
\end{array}\right)=\frac{1}{\sqrt{2}}\left(\begin{array}{c}
\theta_{1}+i \theta_{2} \\
\phi+\delta \phi+i \theta_{3}
\end{array}\right)
$$

\footnotetext{
${ }^{4}$ Note that, contrary to the conformal transformation used in Higgs inflation, the disformal transformation leads to the same Lagrangian eq. (2.7) both in the metric and Palatini formulation of general relativity (at first order in $\varepsilon$ ).

${ }^{5}$ If the Higgs boson is a spectator field during inflation, contrary to what is discussed here, derivative couplings between the inflaton and the Higgs leads to a non-trivial metric in field space that can play a crucial role in determining the fate of the vacuum instability during inflation [40].
} 
with $\phi(t)$ the classical background and $\delta \phi(x, t)$ and $\theta_{i}(x, t)$ the Higgs and Goldstone fluctuations.

From now on we take eq. (2.7) as our starting point. To avoid notational clutter, if there is no chance of confusion we will suppress the flavor indices on the fermion, gauge and Goldstone fields, and on the Yukawa and gauge couplings.

\subsection{Tree level analysis and different regimes}

The action for the classical background Higgs field $\phi$ in eq. (2.9) becomes

$$
\mathcal{L}=\frac{1}{2} m_{\mathrm{P}}^{2} R-\frac{1}{2} K_{\phi}(\phi)\left(\partial_{\mu} \phi\right)^{2}-\frac{\lambda}{4} \phi^{4} .
$$

The dynamics of the system is very different for small and large field values, for which the correction to the Higgs kinetic term is not important respectively dominates. We use the parameter $\delta$ in eq. (2.4) evaluated on the background to parameterize the diffferent regimes:

$$
\text { small field : } \quad \delta \ll 1, \quad \text { large field }: \quad \delta \gg 1 \text {. }
$$

In the small field limit the action reduces to the SM Lagrangian while the large field regime corresponds to the inflationary regime.

Consider the latter and take $\delta \gg 1$. In terms of canonically normalized background field $h$, defined via $\partial h=\sqrt{K_{\phi}} \partial \phi$, the potential becomes

$$
V=\frac{\tilde{\lambda}}{4} m_{\mathrm{P}}^{4}\left(\frac{h}{m_{\mathrm{P}}}\right)^{4 / 3}, \quad \tilde{\lambda}=6^{4 / 3} \lambda^{1 / 3}\left(\mathcal{M} / m_{\mathrm{P}}\right)^{8 / 3} .
$$

which is chaotic inflation with exponent of $4 / 3$ in the potential. It is easy to check that the full period of inflation happens well inside the large field regime. Taking $N_{*}=60$ for the CMB pivot scale, the spectral index and tensor-to-scalar ratio at tree level are

$$
n_{s}=1+\left.\frac{d \ln \mathcal{P}_{R}}{d \ln k}\right|_{\star} \simeq 0.972, \quad r=\left.\frac{\mathcal{P}_{T}}{\mathcal{P}_{R}}\right|_{\star} \simeq 0.089
$$

where $\mathcal{P}_{R}, \mathcal{P}_{T}$ are the scalar and tensor power spectrum and we use the standard singlefield slow-roll approximation that leads to $n_{s \star}-1 \simeq 2 \eta_{V \star}-6 \epsilon_{V \star}$ and $r \simeq 16 \epsilon_{V \star}$ with potential slow-roll parameters defined as $\epsilon_{V}=m_{\mathrm{P}}^{2} / 2\left(V^{\prime} / V\right)^{2}$ and $\eta_{V}=m_{\mathrm{P}}\left(V^{\prime \prime} / V\right)$. The scalar power spectrum fixes the free parameter $M \simeq 1.5 \times 10^{-8} m_{\mathrm{P}} \lambda^{-1 / 4}$. The aim of this paper is to calculate the sensitivity of these results to one-loop RG flow corrections.

\section{Covariant formalism}

We now calculate the RGEs in the inflationary regime. We sketch the methodology and introduce the relevant notation, before presenting the results. The technical details are relegated to appendix A.

The covariant construction is based on the definition of a metric on the field space manifold that is defined by the tensor contained in the highest-derivative term of the 
action [38]. This approach has led Vilkovinsky and de Witt in the Eighties to build the covariant effective action [38, 41], and it is ubiquitous to formulate multi-field dynamics in a covariant form [39, 42-44]. Recently there has been a renewed interest in using geometric covariant formalisms for instance in the context of SMEFT (and extensions) [45-49], to compute UV divergences of general relativity as an EFT [50] and to address the issue of frame equivalence (at quantum level) in scalar-tensor theories [51-53].

Our work aims to provide covariant counterterms to have results that are field reparameterization independent at each step in the derivation of the beta functions. This allows to more systematically include all interactions contributing at a given order, and simplifies calculations considerably.

In truth, once gauge bosons are included, the desired full covariant result is obtained either by using a metric projected on the space of gauge orbits or by using the metric eq. (3.6) but with a specific gauge choice $[54,55]$ different from the Landau gauge used in this work. We consider this delicate issue in detail (and for a more general set-up) in a forthcoming publication that will make it clear that results obtained by means of the two different gauge choices (and the same field space metric in eq. (3.6)) differ by terms that are only higher order in the accuracy used in the large and small field regimes.

\subsection{Large field regime and counterterms}

In the large field regime we can use (2.7) and expand in small $\delta^{-1}$ to capture the dominant effects. At leading order in the $\delta^{-1}$-expansion the mass $\mathcal{M}$ is not an independent parameter, as it can be rescaled from the Lagrangian. Indeed, if we define the tilde fields and couplings via

$$
\tilde{\mathcal{H}}^{6}=\frac{\lambda}{\mathcal{M}^{4}} \mathcal{H}^{6}, \quad \tilde{\lambda}=6^{4 / 3} \lambda^{1 / 3} \mathcal{M}^{8 / 3}, \quad \tilde{y}=y\left(\frac{\mathcal{M}^{4}}{\lambda}\right)^{1 / 6}, \quad \tilde{\alpha}_{0 i}=\alpha_{0 i}\left(\frac{\lambda}{\mathcal{M}^{4}}\right)^{\frac{1}{3}\left(1+\frac{n_{i}}{2}\right)}
$$

the Lagrangian in the large field regime becomes

$$
\begin{aligned}
\mathcal{L}= & -\tilde{\mathcal{H}}^{4}\left|D_{\mu} \tilde{\mathcal{H}}\right|^{2}-6^{-4 / 3} \tilde{\lambda}|\tilde{\mathcal{H}}|^{4}-\left(\frac{\tilde{y}}{\sqrt{2}} \bar{q}_{L} \mathcal{H}_{c} t_{R}+\text { h.c. }\right) \\
& -\sum_{a} \frac{1}{4} \tilde{\alpha}_{0 A}|\tilde{\mathcal{H}}|^{4\left(1+\frac{n_{A}}{2}\right)}\left(F_{\mu \nu}^{a}\right)^{2}+\sum_{i} \tilde{\alpha}_{0 F}|\tilde{\mathcal{H}}|^{4\left(1+\frac{n_{F}}{2}\right)} \bar{\psi}_{i}(i \not D) \psi_{i}+\mathcal{O}\left(\delta^{-1}\right) .
\end{aligned}
$$

Also in the small field regime, where the model reduces to the SM, the scale $\mathcal{M}$ drops out of the Lagrangian at leading order in the $\delta$-expansion. The scale $\mathcal{M}$ cannot be removed over the whole field range though. In fact, it still plays a fundamental role in determining the boundaries between the small and large field region and the matching conditions between the parameter of the two (see section 5.2).

We will calculate the loop corrections in the untilde variables, as this makes the $\delta^{-1}$ -expansion more transparent. As just shown, the original variables are not all independent at first order in the $\delta^{-1}$ expansion, and the resulting counterterms will form a system that 
is not closed. ${ }^{6}$ We then translate the counterterms to those for the set of independent tilde-variables (3.4) to derive the beta functions for the tilde variables.

Counterterms are introduced in the usual way by rescaling the bare fields and couplings by $Z_{i}=1+\delta_{i}$, with $\delta_{i}$ the counterterm:

$$
\begin{aligned}
& \mathcal{H}_{b}=\sqrt{Z_{\phi}} \mathcal{H}, \quad \psi_{b}=\sqrt{Z_{\psi}} \psi, \quad A_{b}^{\mu}=\sqrt{Z_{A}} A^{\mu}, \quad \alpha_{b}=Z_{\alpha} \alpha, \\
& \lambda_{b}=Z_{\lambda} \lambda, \quad \mathcal{M}_{b}=Z_{\mathcal{M}} \mathcal{M}, \quad y_{b}=Z_{y} y, \quad g_{b}=Z_{g} g \text {. }
\end{aligned}
$$

The relation to the tilde counterterms follows from the rescaling relation eq. (3.1) and is

$$
Z_{\tilde{\phi}}^{3}=Z_{\lambda} Z_{\mathcal{M}}^{-4} Z_{\phi}^{3}, \quad Z_{\tilde{\lambda}}=Z_{\lambda}^{1 / 3} Z_{\mathcal{M}}^{8 / 3}, \quad Z_{\tilde{y}}=Z_{y} Z_{\mathcal{M}}^{2 / 3} Z_{\lambda}^{-1 / 6}, \quad Z_{\tilde{\alpha}_{i}}=Z_{\alpha_{i}}\left(Z_{\lambda} Z_{\mathcal{M}}^{-4}\right)^{\frac{1}{3}\left(1+\frac{n_{i}}{2}\right)} .
$$

To extract the beta functions only the divergent part of the one-loop corrections is needed. We will calculate the two-point functions using dimensional regularization where we drop all finite contributions and only keep the $\epsilon$-poles. The one-loop beta functions for the couplings and anomalous dimensions are then extracted from the counterterms via

$$
-\partial_{t}\left(\ln Z_{\lambda_{i}}\right)=\lim _{\epsilon \rightarrow 0} \epsilon\left(Z_{\lambda_{i}}-1\right)=\frac{\beta_{\lambda_{i}}}{\lambda_{i}}, \quad \frac{1}{2} \partial_{t}\left(\ln Z_{\phi}\right)=-\frac{1}{2} \lim _{\epsilon \rightarrow 0} \epsilon\left(Z_{\phi}-1\right)=\gamma_{\phi},
$$

with $t=\ln \mu, \mu$ the renormalization scale and $\epsilon=4-d$.

\subsection{Covariant fields}

The Higgs field is decomposed in background plus perturbations as in eq. (2.9). We work in Landau gauge (see eq. (A.3)) for which the ghosts fields decouple. The bosonic fields can be grouped together $\varphi^{I}=\phi^{I}+\delta \phi^{I}=\left\{\varphi^{a}, A_{\mu}^{i}\right\}$, with $a$ running over the Higgs and Goldstone fields $\varphi^{a}=\left\{\varphi, \theta_{1}, \theta_{2}, \theta_{3}\right\}$, and $i$ running over the SM gauge fields. The field space manifold has a non-trivial geometry defined by the metric

$$
G_{I J}=\left\{K_{\phi}\left(\varphi^{I}\right) \delta_{a b}, K_{A_{j}}\left(\varphi^{I}\right) \eta_{\mu \nu} \delta_{i j}\right\}=\left\{(1+\delta) \delta_{a b},\left(1+\alpha_{A} \delta\right) \eta_{\mu \nu} \delta_{i j}\right\}
$$

Because the fluctuations $\delta \phi^{I}$ are not covariant objects on the field space manifold, one has to deal with intermediate results (for the counterterms and the effective action) that are not covariant under fields reparameterizations. This makes it hard to organize the calculation and include all relevant interactions. For example, it is well known that the one-loop Coleman-Weinberg potential depends on the covariant mass matrix of the bosonic fields running in the loop $m_{J}^{I}=G^{I K} \nabla_{K} \nabla_{J} V$, with $\nabla_{I}$ the covariant derivative constructed from the field space metric in eq. (3.6). To obtain this result expanding the action in $\delta \phi^{I}$ requires using the background equations of motion as well.

To expand the Lagrangian in a form that is fully covariant under field redefinitions one should replace the ordinary field displacement $\varphi^{I}-\phi^{I}$ with the tangent vector to the unique geodesic connecting the background $\phi^{I}$ to the field $\varphi^{I}$

$$
\varphi^{I}-\phi^{I} \rightarrow Q^{I}=\left.\frac{d \varphi^{I}(\tau)}{d \tau}\right|_{\tau=0},
$$

\footnotetext{
${ }^{6}$ If one tries to find the running of $\mathcal{M}$ (and the other original parameters in the Lagrangian), there are not enough conditions to solve for all counterterms and derive all beta functions.
} 
where $\tau$ is the affine parameter parameterizing the geodesic such that $\varphi^{I}(\tau=1)=\varphi^{I}$ and $\varphi(0)=\phi^{I}$. Let us summarize the notations in the following table

\begin{tabular}{|c|c|c|}
\hline $\mathrm{I}$ & Non-covariant fields $\delta \phi^{I}$ & Covariant fields $Q^{I}$ \\
\hline$\phi$ & \multicolumn{2}{|c|}{$\delta \phi=Q^{\phi}+\mathcal{O}\left(Q^{2}\right)$} \\
\hline$\theta$ & \multicolumn{2}{|c|}{$\theta=Q^{\theta}+\mathcal{O}\left(Q^{2}\right)$} \\
\hline A & \multicolumn{2}{|c|}{$A_{\mu}=Q^{A_{\mu}}+\mathcal{O}\left(Q^{2}\right)$} \\
\hline
\end{tabular}

In order to find the relation between the non-covariant displacements $\delta \phi^{I}$ and the covariant ones $Q^{I}$, one can expand $\delta \phi^{i} \equiv \varphi^{i}-\phi^{i} \equiv \varphi^{i}(\tau=1)-\varphi^{i}(\tau=0)$ in Taylor series around zero in the affine parameter and recursively use the geodesic equation satisfied by $d \varphi^{I} / d \tau$; this gives

$$
\delta \phi^{i}=\left.\sum_{n=0}^{\infty} \frac{1}{n !} \frac{d^{n} \varphi^{i}}{d \tau^{n}}\right|_{0}=Q^{i}-\frac{1}{2} \Gamma_{j k}^{i} Q^{j} Q^{k}+\frac{1}{3 !}\left(\Gamma_{l m}^{i} \Gamma_{j k}^{m}-\partial_{l} \Gamma_{j k}^{i}\right) Q^{j} Q^{k} Q^{l}+\ldots
$$

where $\Gamma_{I J}^{K}$ are the Christoffel symbols associated to the metric $G_{I J}$ evaluated on the background. To expand the action in covariant form we can consider $S(\varphi)$ as a function of the affine parameter $\tau$ evaluated in $\tau=1$, i.e.

$$
S(\varphi)=\left.\sum_{n=0}^{\infty} \frac{1}{n !} \frac{d^{n} S}{d \tau^{n}}\right|_{\tau=0}=\sum_{n=0}^{\infty} \frac{1}{n !} Q^{i_{1}} \cdots Q^{i_{n}}\left[\nabla_{\left(i_{1}\right.} \cdots \nabla_{\left.i_{n}\right)} S\right][\phi],
$$

where we used $\frac{d}{d \tau} \equiv \frac{d \varphi^{i}}{d \tau} \nabla_{i}$ and the geodesic equation. The round brackets mean symmetrization over the indices. The coefficients of the expansion are evaluated on the background and will determine the strength of the interactions. In particular, we expand in this way the scalar functions $\left\{V, K_{\phi}, K_{\psi}, K_{A}\right\}$ and the Yukawa and gauge interactions. Equivalently, the action can be expanded by normal Taylor series in the fluctuations $\delta \phi^{I}$, and then substitute their expression in terms of the covariant fields given in eq. (3.9). ${ }^{7}$ We use this second approach to expand the kinetic terms in covariant fluctuations. In this expansion we neglect terms with time derivative of the background ( $\dot{\phi}^{2}$-corrections), as well as the backreaction from gravity, which are both slow-roll suppressed during inflation.

\section{Renormalization group flow}

In this section we calculate the one-loop beta functions for SM with non-minimally derivative couplings eq. (2.7). In the small field regime the set-up reduces to the SM EFT with the SM RGEs to leading order in the $\delta$-expansion. In the large field inflationary regime, the EFT can be expanded in $\delta^{-1}$. As we show, the EFT is renormalizable in the sense that all divergences can be absorbed in counterterms order by order in the $\delta^{-1}$-expansion.

\footnotetext{
${ }^{7}$ The two procedures give the same results since both represent the same expansion of the action in the affine parameter $\tau$.
} 


\subsection{Renormalization group equations}

We compute one-loop corrections to the Higgs, Goldstone boson, fermion and gauge boson two-point functions, and expand in $\delta^{-1}$ to find the leading order contribution in the large field regime. This gives the various counterterms in the theory, and consequently the beta functions using eq. (3.5). The idea is to provide a systematic procedure to compute one-loop beta functions in similar set-ups. Let us remind that we calculate the quantum corrections in the untilde variables, as this makes the $\delta^{-1}$-expansion more transparent. However, we rewrite the results in terms of the independent (and relevant) set of couplings, for which we derive the RGEs.

The momentum dependent part of the two-point functions gives the counterterm for the kinetic terms, whereas the momentum independent part provides the counterterm for the two-point vertexes. We will denote these with $Z_{2 f}$ and $Z_{c 2 f}$ respectively, with $f=\left\{Q^{\phi}, Q^{\theta}, Q^{A}, \psi\right\}$ the (covariant) fields in question, and $c=\{\lambda, g, y\}$ if it renormalizes the Higgs, gauge or Yukawa coupling. The relevant counterterms are those of the quadratic Lagrangian. These $Z$-factors can be expressed in terms of the basis set of counterterms introduced in (3.3). For example, from the Higgs kinetic term in the large field regime

$$
\mathcal{L} \supset \frac{1}{2} Z_{\phi}^{2} Z_{\lambda} Z_{\mathcal{M}}^{-4} Z_{\phi} \delta\left(\partial Q^{\phi}\right)^{2} \equiv \frac{1}{2} Z_{2 Q^{\phi}} \delta\left(\partial Q^{\phi}\right)^{2} .
$$

The full set of $Z$-relations in the large field regime and at leading order is given in eq. (A.9).

To understand the results given in the next subsections it is useful to look at the masses of the various particles. This allows to determine which particles have masses of the inflationary scale and are included in the EFT spectrum for different choices of the nonminimal couplings, and which are too heavy or too light (too weakly coupled) to contribute to the loop corrections at leading order. The masses of the gauge and fermion fields depend on the functions $K_{A}$ and $K_{\psi}$ in their kinetic terms. The masses of the bosonic fields are given by the covariant expression $\left(m^{2}\right)_{I}^{J}=-G^{I J} \nabla_{I} \nabla_{J} \mathcal{L}$ evaluated on the background:

$$
m_{h}^{2}=\lambda \phi^{2} \frac{(3+\delta)}{(1+\delta)^{2}}, \quad m_{\theta}^{2}=\lambda \phi^{2} \frac{(1+3 \delta)}{(1+\delta)^{2}}, \quad m_{A}^{2}=\frac{g_{i}^{2} \phi^{2}(1+\delta)}{\left(1+\alpha_{A} \delta\right)}+\frac{\delta\left(2+n_{A}\right) \alpha_{A} \lambda \phi^{2}}{(1+\delta)\left(1+\alpha_{A} \delta\right)},
$$

with $n_{A}$ determining the non-minimal gauge coupling (2.5). We used the notation $\left(m^{2}\right)_{Q^{a}}^{Q^{a}} \equiv$ $m_{a}^{2}$. The last term in the gauge boson mass arises from mixing between the Higgs and gauge sector (specifically, because $\Gamma_{A A}^{\phi} \neq 0$ ), and it is suppressed at large field values for $n_{A}<1$. In principle we should also define covariant fermion fluctuations, but it is not clear how to do that rigorously. We can find a parametric estimate of the fermion mass by rescaling $K_{\psi}(\phi) \psi \rightarrow \psi$ to obtain approximately canonically renormalized fermions, where we evaluate the function $K_{\psi}$ on the background. This gives

$$
m_{\psi} \sim \frac{1}{\sqrt{2}} K_{\psi}^{-1} y_{i} \phi
$$

Below we will summarize the results for the two-point functions and beta functions for the various cases defined in eq. (2.5). The technical details and the Feynman rules are given in appendix A. 


\subsubsection{Case A}

Let us start with case A. The top quark and gauge bosons are minimimally coupled and have standard kinetic terms for $\alpha_{0 f}=\alpha_{0 A}=0$. The Higgs and Goldstone are light $m_{h, \theta}^{2} \sim \mathcal{O}\left(\delta^{-1}\right) V^{1 / 2}$, and their fluctuations decouple; this holds for all the cases we discuss. The top quark has mass $m_{t}^{2} \sim V^{1 / 2}$ and is in the spectrum. The gauge bosons on the other hand are heavy $m_{A}^{2}=\mathcal{O}(\delta) V^{1 / 2}$ and should be integrated out; to obtain a renormalizable EFT at lower energies requires new physics at this mass scale, as the gauge field loop contribution is non-renormalizable [15]. ${ }^{8}$

The one-loop expressions for the self-energies are given in appendix A.4-A.5. The counterterms for the Higgs and fermion kinetic terms and for the fermion two-point interaction vanish, while the Higgs two-point vertex gets a corrections from the top loop:

$$
Z_{2 Q^{\phi}}=Z_{2 \psi}=Z_{y 2 \psi}=1+\mathcal{O}\left(\delta^{-1}\right), \quad Z_{\lambda 2 Q^{\phi}}=1+\boldsymbol{A}+\mathcal{O}\left(\delta^{-1}\right)
$$

with

$$
\boldsymbol{A}=-\frac{1}{8 \pi^{2} \epsilon}\left(\frac{N_{c} y^{4}}{\lambda}\right)=-\frac{1}{8 \pi^{2} \epsilon}\left(\frac{6^{4 / 3} \tilde{y}^{4}}{\tilde{\lambda}}\right) .
$$

with $N_{c}=3$ the number of colors.

We can understand these results parameterically by setting $K_{\phi}$ to its constant background value, and evaluting diagrams with the unrenormalized Higgs field $\delta \phi$ and fermion fields on the external lines. The effect of $K_{\phi} \sim \delta$ is that the Higgs/Goldstone propagator is suppressed by a factor $\delta^{-1}$, the Higgs-gauge couplings (which reside inside the kinetic terms) enhanced by a factor $\delta$, and the diagram with a $Z_{2 Q^{\phi}}$ counterterm is also enhanced by a factor $\delta$. Thus Higgs loops are suppressed, and since the gauge fields are integrated out, there is no leading order contribution to the fermion self-energy. The top loop contribution to the Higgs two-point vertex is as in the SM, however the wave function correction $Z_{2 Q^{\phi}} \propto \delta^{-1}$ is suppressed.

The beta functions are derived using (3.5), and depend on the logarithm of the $Z$ factors. We are thus interested in

$$
\begin{aligned}
& 0=\ln \left(Z_{2 Q^{\phi}}\right)=\ln \left(Z_{\tilde{\phi}}^{3}\right), \\
& 0=\ln \left(Z_{y 2 \psi}\right)=\ln \left(Z_{\psi} Z_{\tilde{y}} Z_{\tilde{\phi}}^{1 / 2}\right), \quad \ln (1+\boldsymbol{A})=\ln \left(Z_{\lambda 2 Q^{\phi}}\right)=\ln \left(Z_{\tilde{\lambda}} Z_{\tilde{\phi}}^{2}\right),
\end{aligned}
$$

where in the second step we used the relation between different counterterms (see eq. (A.9) in the appendix) and (3.4). Note that $Z_{\mathcal{M}}$ has dissapeared when written in terms of the tilde-variables, as it should. We can solve this system of equations to get

$$
Z_{\tilde{\phi}}=Z_{\psi}=Z_{\tilde{y}}=1, \quad Z_{\tilde{\lambda}}=1+\boldsymbol{A} .
$$

The beta functions are then

$$
\left(\beta_{\tilde{y}}, \gamma_{\psi}, \gamma_{\tilde{\phi}}\right)=\mathcal{O}\left(\delta^{-1}\right), \quad \beta_{\tilde{\lambda}}=-\frac{1}{8 \pi^{2}} 6^{4 / 3} N_{c} \tilde{y}^{4} .
$$

${ }^{8}$ The loop-correction to the Higgs two-point vertex can be calculated in the $g \ll 1$ limit, such that the gauge boson mass is below the cutoff scale of the theory. It scales with $\delta^{2}$ and is thus large, and it cannot be absorbed in the counterterms of the Lagrangian. 


\subsubsection{Case B}

Consider non-minimal kinetic terms for the gauge fields $K_{A}=1+\alpha_{0} \delta$, which brings them back in the spectrum during inflation $m_{A}^{2} \sim V^{1 / 2}$. The counterterms now become

$$
\begin{aligned}
Z_{2 Q^{\phi}} & =Z_{\psi}=Z_{m_{\psi}}=Z_{2 Q^{A}}=Z_{g 2 Q^{A}}=1+\mathcal{O}\left(\delta^{-1}\right), \\
Z_{\lambda 2 Q^{\phi}} & =Z_{\lambda 2 Q^{\theta}}=Z_{V}=1+\boldsymbol{A}
\end{aligned}
$$

with

$$
\boldsymbol{A}=\frac{1}{8 \pi^{2} \epsilon} \frac{1}{\lambda}\left(\sum_{i} \frac{3 g_{i}^{4}}{\alpha_{0}^{2}}-N_{c} y^{4}\right)=\frac{1}{8 \pi^{2} \epsilon} \frac{6^{4 / 3}}{\tilde{\lambda}}\left(\sum_{i} \frac{3 g_{i}^{4}}{\tilde{\alpha}_{0}^{2}}-N_{c} \tilde{y}^{4}\right) .
$$

The summation is over the massive electroweak gauge bosons, the $W^{ \pm}$bosons and the $Z$-boson, with couplings $g_{i}=\frac{1}{2} \times\left\{g, g, \sqrt{g^{2}+g^{\prime 2}}\right\}$, and $g$ and $g^{\prime}$ the $\mathrm{SU}(2)$ and $\mathrm{U}(1)$ gauge coupling respectively.

The counterterms derived from the Higgs self-energy are consistent with those derived from the Goldstone self-energy, and also from the effective potential given in [15]. However, it is interesting to note that only by including some of the genuinely new interactions coming from the $Q$-expansion of the gauge fields (that are absent for canonical gauge fields), we are able to find agreement for the various counterterms. Particularly important are the interactions $^{9}$

$$
\mathcal{L}_{\mathrm{k}}=-\frac{1}{2} G_{I J}\left(\varphi^{I}\right) \partial \varphi^{I} \partial \varphi^{J} \supset-\mathcal{K}_{2 Q^{\phi} 2 \partial Q^{I}}\left(Q^{\phi}\right)^{2}\left(\partial Q^{I}\right)^{2}-\mathcal{K}_{2 Q^{\theta} 2 \partial Q^{I}}\left(Q^{\theta}\right)^{2}\left(\partial Q^{I}\right)^{2}
$$

where $\mathcal{K}_{2 Q^{I} \partial Q^{J}}$ are the background dependent coefficients given by expanding the noncanonical kinetic term in covariant fields. For example $\mathcal{K}_{2 Q^{\phi} 2 \partial Q^{A}}=\alpha_{A 0} \delta\left\{0,-1 / 3 \phi^{2}\right\}$ where the terms in curly brackets give the leading terms in the SM regime and large field regime respectively. These new interactions give a contribution to the Higgs self-energy

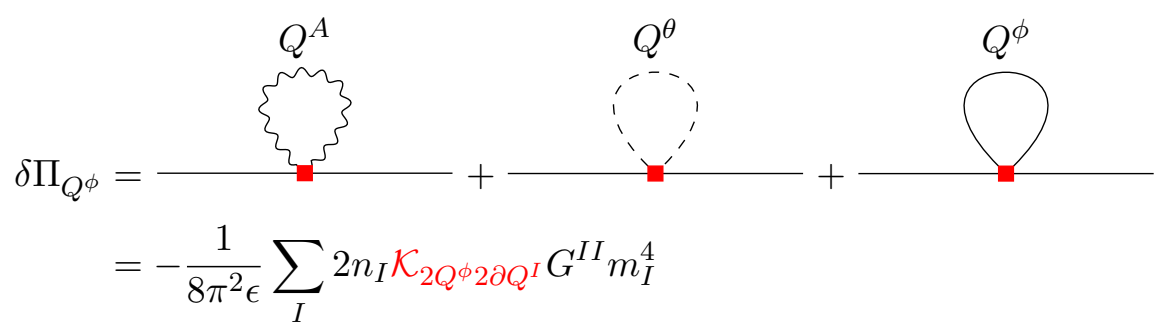

and similar for the Goldstone boson self-energy. Here the sum is over $Q^{I}=\left\{Q^{\phi}, Q^{\theta}, Q^{A_{\mu}}\right\}$ with $n_{I}=\{1,3,4 \times 3\}$ the d.o.f. Only the gauge boson contributes at leading order, as the Higgs/Goldstone mass is suppressed during inflation. Including this correction, see appendix A.2 for more details, the results for all counterterms are consistent.

We can once again understand the results in eq. (4.10) parametrically, by taking $K_{\phi}, K_{A} \propto \delta$ on the background. The difference with case $\mathrm{A}$ is that now also the gauge boson propagator is suppressed by $\delta^{-1}$, the gauge non-abelian self-interactions (which reside inside the gauge kinetic terms) enhanced by a factor $\delta$, and the diagram with a $Z_{2 Q^{A}}$

\footnotetext{
${ }^{9}$ The couplings $\mathcal{K}_{Q^{\phi} 2 \partial Q^{I}}=\mathcal{K}_{Q^{\theta} 2 \partial Q^{I}}=0$ vanish
} 
counterterm is also enhanced by a factor $\delta$. Remembering that the gauge-Higgs couplings are enhanced, we see that now the gauge loop does contribute to the Higgs self-energy at leading order as the relevant diagrams scale with powers of $\left(K_{\phi} / K_{A}\right)=\mathcal{O}(1)$. There is no leading order contribution to $Z_{2 Q^{A}}$. This is because both the diagrams with a gauge-loop (the enhancement of the gauge-interactions is cancelled by the suppressed gauge boson propagator) and fermion loop (the fermion interactions are standard) are $\mathcal{O}(1)$, while the diagram with the counterterm is enhanced. There is also no gauge boson contribution to the fermion self-energy as the corresponding diagrams are suppressed by the gauge-boson propagator.

The Higgs and fermion $Z$-factors give the same results as in case A given in (4.6), except that $\boldsymbol{A}$ now includes the gauge contribution. In addition, the gauge interactions give

$$
\begin{aligned}
& 0=\ln \left(Z_{2 Q^{A}}\right)=\ln \left(\frac{Z_{\tilde{\alpha}_{0}} Z_{\tilde{\phi}}^{2}}{Z_{g}^{2}}\right), \\
& 0=\ln \left(Z g_{2 Q^{A}}\right)=\ln \left(Z_{\tilde{\phi}}^{3}\right)
\end{aligned}
$$

where we used the Ward identity $Z_{g_{i}}^{2} Z_{A_{i}}=1$ (no summation). We can solve the system of equations to get

$$
Z_{\tilde{\phi}}=Z_{\psi}=Z_{\tilde{y}}=\frac{Z_{g}^{2}}{Z_{\tilde{\alpha}_{0}}}=1+\mathcal{O}\left(\delta^{-1}\right), \quad Z_{\tilde{\lambda}}=1+A
$$

The beta functions are then

$$
\beta_{\tilde{y}}=\beta_{g^{2} / \tilde{\alpha}_{0}}=\gamma_{\psi}=\gamma_{\tilde{\phi}}=\gamma_{A}=O\left(\delta^{-1}\right), \quad \beta_{\tilde{\lambda}}=\frac{1}{8 \pi^{2}} 6^{4 / 3}\left(3 \sum_{i} \frac{g_{i}^{4}}{\tilde{\alpha}_{0}^{2}}-N_{c} \tilde{y}^{4}\right)
$$

\subsubsection{Case $\mathrm{C}$ and $\mathrm{D}$}

Case $\mathrm{C}$ is analogous to case $\mathrm{B}$ with the only difference that this time the fermion is light and decouples. Thus $\beta_{\tilde{\lambda}}=1 / 8 \pi^{2} 6^{4 / 3}\left(\sum_{i} 3 g_{i}^{4} / \tilde{\alpha}_{0}^{2}\right)$. In case D all fields are weakly coupled, $\beta_{\tilde{\lambda}}=\mathcal{O}\left(\delta^{-1}\right)$, and nothing runs.

\subsection{Beta-functions summary}

The RGEs during inflation can be summarized as follows

$$
\begin{aligned}
& \beta_{\tilde{y}}=\beta_{g^{2} / \tilde{\alpha}_{0}}=\gamma_{\psi}=\gamma_{\tilde{\phi}}=\gamma_{A}=\mathcal{O}\left(\delta^{-1}\right), \\
& \beta_{\tilde{\lambda}}=\frac{1}{8 \pi^{2}} 6^{4 / 3}\left(3 f_{1} \sum_{i} \frac{g_{i}^{4}}{\tilde{\alpha}_{0}^{2}}-f_{2} 3 \tilde{y}^{4}\right),
\end{aligned}
$$

with $g_{i}=\frac{1}{2}\left\{g, g, \sqrt{g^{2}+g^{\prime 2}}\right\}$, and $g$ and $g^{\prime}$ the $\mathrm{SU}(2)$ and $\mathrm{U}(1)$ gauge coupling respectively. The $f_{i}$ take on a value of zero/one depending on the non-minimal couplings of the 
fermion/gauge fields, specifically

$$
\begin{aligned}
& \text { Case A : } \quad\left(f_{1}, f_{2}\right)=(0,1), \\
& \text { Case B : } \quad\left(f_{1}, f_{2}\right)=(1,1), \\
& \text { Case C : } \quad\left(f_{1}, f_{2}\right)=(1,0), \\
& \text { Case D : } \quad\left(f_{1}, f_{2}\right)=(0,0) .
\end{aligned}
$$

\section{Predictions for inflation}

We are now in the position to calculate corrections to the inflationary observables $n_{s}$ and $r$ due to the running of the couplings.

For the boundary values of the couplings at the electroweak scale we use the twoloop matching conditions of [56]. ${ }^{10}$ The inflationary parameters are sensitive to the top and Higgs masses $\left(m_{t}^{\mathrm{EW}}, m_{h}^{\mathrm{EW}}\right) \equiv\left(M_{t}, M_{h}\right)$ and the strong coupling constant $\alpha_{s}$. The top mass determination gives the largest uncertainty, both from an experimental and a theoretical point of view (see $[57,58]$ for recent discussions). The best current estimate is $M_{t}=172.9 \pm 0.4 \mathrm{GeV}$ [59]. We fix, for illustrative purposes, $\alpha_{s}$ to its central value $\alpha_{s}=0.1181$ and $M_{h}=125.6 \mathrm{GeV}$ [59].

In the small field regime $(\delta<1)$ we use the SM two-loop beta functions [56, 60] to compute the running of the SM parameters $\{\lambda, y, \ldots\}$. At the boundary $\delta=1$ we match to the rescaled couplings $\{\tilde{\lambda}, \tilde{y}, \ldots\}$ of the large field regime $(\delta>1)$, and use the one-loop beta functions derived in the previous section for their running. As already mentioned, the corrections due to unknown UV physics on the running gives rise to threshold corrections which we parameterize, following the approach in $[11-13,15]$, by a jump in the couplings at the boundary between the two regimes.

We restrict to the tree-level $\mathrm{RG}$ improved potential for our numerical analysis since we have derived only the one-loop beta functions in the large field regime.

\subsection{Renormalization group dependence: analytical estimate}

Before turning to the actual numerical implementation we quickly recap the analytical estimate of the effect of the running on the inflationary observables [15]. With the explicit form of the RGEs we can now verify some of the assumptions previously made.

We consider the RG improved effective action in terms of the canonical field $h$, see eqs. (2.12) and (3.1)

$$
h=\frac{\sqrt{\lambda} \phi^{3}}{6 \mathcal{M}^{2}}=\frac{\tilde{\phi}^{3}}{6}
$$

frow which it follows that $\gamma_{h}=3 \gamma_{\tilde{\phi}}$. In all cases A-D considered in the previous section we found $\gamma_{\tilde{\phi}}=\mathcal{O}\left(\delta^{-1}\right)$, and we can thus neglect the anomalous dimension since $\gamma_{h}=$

\footnotetext{
${ }^{10}$ The instability of the Higgs potential (when $\lambda(\mu)$ becomes negative) is pushed to larger scales with two-loop matching conditions compared to one-loop matching.
} 
$\mathcal{O}\left(\delta^{-1}\right), \gamma_{h}^{\prime}=\mathcal{O}\left(\delta^{-1}\right) .{ }^{11}$ The leading-order RG improved action then becomes

$$
\mathcal{L}=\frac{1}{2} R-\frac{1}{2} \partial_{\mu} h \partial^{\mu} h-\frac{\tilde{\lambda}(t)}{4} h^{4 / 3} .
$$

The RG improved potential is shown in figure 2a for different boundary conditions of the top mass and for different threshold conditions paramerized by a jump $\Delta \lambda$ in the Higgs coupling at the matching scale. For large top mass and/or large negative jump the potential develops a maximum.

We choose as renormalization scale the top mass. The RG time is then (using again eq. (3.1))

$$
t=\ln \left(\frac{\mu}{m_{\mathrm{t}}^{\mathrm{EW}}}\right), \quad \mu=y \phi=\tilde{y} \tilde{\phi}=\tilde{y} 6^{1 / 3} h^{1 / 3} .
$$

Often the Yukawa coupling is neglected for simplicity as $y=\mathcal{O}(1)$. However, one should keep $\tilde{y}$ explicitly as it can be small. Indeed, using tree-level relations derived below eq. (2.13) for e.g. $y \simeq 10^{-1}$ and $\lambda \simeq 10^{-4}$ we find $\tilde{y} \simeq 10^{-4}$. Running effects enter the observables, because calculating slow-roll parameters (derivatives of the potential) also involves taking the derivative of the $\tilde{\lambda}$-coupling in the potential [15]:

$$
\frac{d \tilde{\lambda}(\mu)}{d h} \equiv \beta_{\tilde{\lambda}} \frac{d t}{d h}=\frac{\beta_{\tilde{\lambda}}}{3 h}+\mathcal{O}\left(\delta^{-1}\right), \quad \frac{d \beta_{\tilde{\lambda}}}{d t} \equiv \beta_{\tilde{\lambda}}^{\prime}=\mathcal{O}\left(\delta^{-1}\right),
$$

where we used the RGEs summarized in eq. (4.17). When the top quark decouples and the gauge boson remains in the spectrum, as in case $\mathrm{C}$, the gauge boson mass is the appropiate scale and the same equations hold with $\tilde{y}_{t}$ replaced by $g / \tilde{\alpha}_{0}$. The potential slow-roll parameters are $\epsilon_{V}=8 /\left(9 h^{2}\right)\left(1+\beta_{\tilde{\lambda}} / 4 \tilde{\lambda}\right)^{2}$ and $\eta_{V}=4 /\left(9 h^{2}\right)\left(1+5 \beta_{\tilde{\lambda}} / 4 \tilde{\lambda}\right)$. The number of $e$-folds before the end of inflation is $N_{\star} \approx 3 h_{\star}^{2} D_{\star} / 8$, where we assumed $^{12}$

$$
D_{\star}=\left(1+\frac{\beta_{\tilde{\lambda}}}{4 \tilde{\lambda}}\right)_{\star}^{-1} \approx \text { constant } \Rightarrow \frac{\beta_{\tilde{\lambda}}}{\tilde{\lambda}} \ll\left(\frac{\beta_{\tilde{\lambda}}}{\tilde{\lambda}}\right)^{2} .
$$

To leading order in the $1 / N_{\star}$ expansion the observables become

$$
n_{s}-1 \simeq-\frac{5}{3 N_{\star}}\left(1+\frac{2}{5} \frac{\beta_{\tilde{\lambda}}}{4 \tilde{\lambda}}\right)_{\star}, \quad r \simeq \frac{16}{3 N_{\star}}\left(1+\frac{\beta_{\tilde{\lambda}}}{4 \tilde{\lambda}}\right)_{\star} .
$$

The influence of the RG flow can become significant if the ratio $\beta_{\tilde{\lambda}} /(4 \tilde{\lambda})$ is order one during inflation.

\subsection{Matching and running: numerical results for case $\mathrm{A}$}

The linear analysis of the previous section indicates an RG dependence of the inflationary observables. We now compute numerically the size of these corrections, starting with

\footnotetext{
${ }^{11}$ As a consequence, our results for slow-roll parameters and observables are implicitly formulated in a gauge-invariant fashion as in [61].

${ }^{12}$ Note that, in contrast to the case of the Cosmological Attractors (see [29]), one cannot consider the beta functions dependent factor $D$ constant over the integration domain when computing $N_{*}$ as a function of the field. In fact, expanding the integrand, all the terms of the series contribute at the same order in the small parameter $\delta^{-1}$.
} 

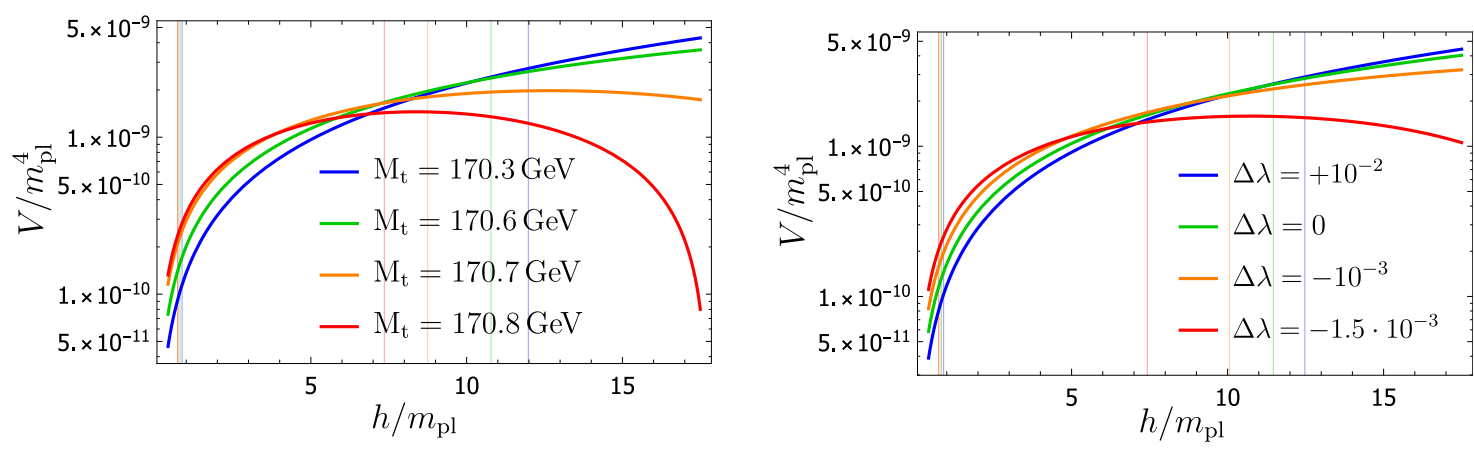

(a) RG improved potentials as a function of the canonical inflaton field $h$. The left (right) vertical lines correspond (for each scenario) to the beginning (end) of inflation.
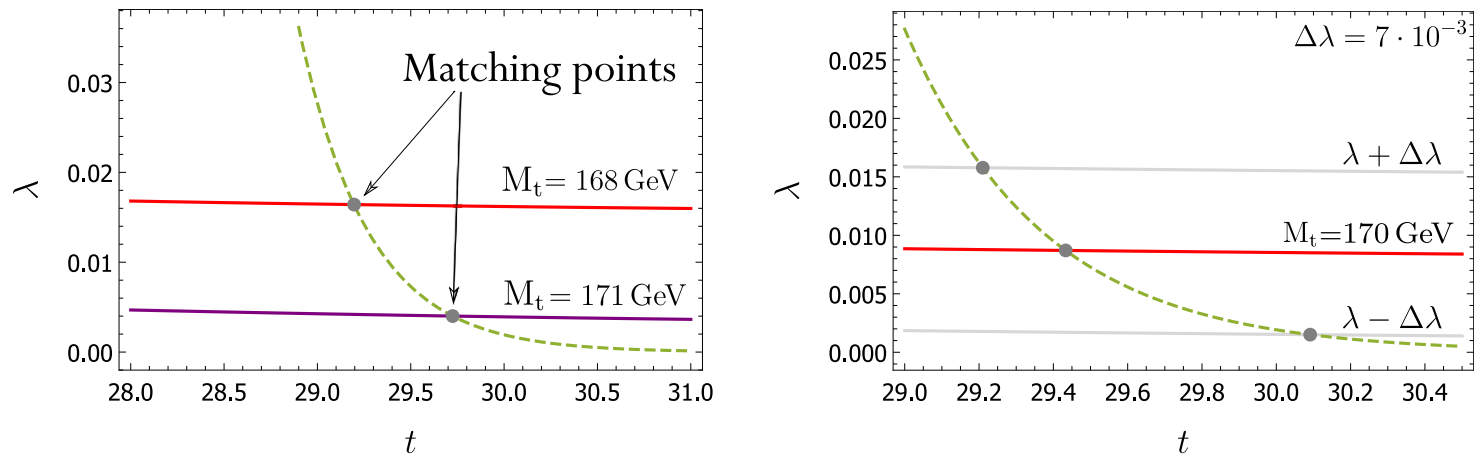

(b) Intersection of the running coupling $\lambda(t)$ with the curve $\alpha e^{-8 t / 3}$ (dashed green line) from (5.15) as a function of the renormalization time $t$. The intersection points give $\left(t_{\text {eq }}, \lambda_{\text {eq }}\right)$ and define the matching point. A larger top mass/negative threshold correction implies a smaller $\lambda_{\text {eq }}$, and consequently larger corrections to the observables (see eq. (5.16)).

Figure 2. Case A: RG improved potential and matching point for $M_{h}=125.6 \mathrm{GeV}$ and different top mass (left) and $\left(M_{h}, M_{t}\right)=(125.6,170) \mathrm{GeV}$ and different threshold corrections (right).

case A. In the small field regime we run the SM parameters $\{\lambda, y, \ldots\}$ using the 2-loop SM beta functions $[56,60]$, and with boundary condtions at the EW scale. At the border between the two regimes

$$
\left.\delta\right|_{t_{\mathrm{eq}}}=1 \Longrightarrow \phi_{\mathrm{eq}}=\left(\frac{4 \mathcal{M}^{4}}{\lambda\left(t_{\mathrm{eq}}\right)}\right)^{1 / 4}
$$

we switch to the rescaled couplings $\{\tilde{\lambda}, \tilde{y}, \ldots\}$. Threshold corrections can be incorporated (and parameterized) by a jump in the coupling constants at $t_{\text {eq }}$. Beyond the matching point we run with the one-loop beta functions valid in the large field regime eq. (4.17). The power spectrum constraint fixes

$$
P_{\mathcal{R}}=2 \cdot 10^{-9} \Longrightarrow \tilde{\lambda}_{\star} \equiv \tilde{\lambda}\left(t_{\star}\right)=4 \cdot 10^{-10},
$$

where $t_{\star}$ is as usual the value of the RG time eq. (5.3) at horizon crossing.

The large field RGEs (4.17), (4.18) for case A are

$$
\beta_{\tilde{y}} \approx 0, \quad \frac{\beta_{\tilde{\lambda}}}{\tilde{\lambda}}=-\frac{3 \times 6^{4 / 3}}{8 \pi^{2}} \frac{\tilde{y}^{4}}{\tilde{\lambda}} .
$$


The first equation trivially implies $\beta_{\tilde{\lambda}}^{\prime} \approx 0$. The matching conditions at the boundary depend on the scale $\mathcal{M}$

$$
\tilde{\lambda}_{\text {eq }}=6^{4 / 3} \lambda_{\text {eq }}^{1 / 3} \mathcal{M}^{8 / 3}, \quad \tilde{y}_{\text {eq }}=y_{\text {eq }} \lambda_{\text {eq }}^{-1 / 6} \mathcal{M}^{2 / 3},
$$

where we used (3.1), and we introduced the notation $X\left(t_{\text {eq }}\right) \equiv X_{\text {eq }}$. We can now understand how the predictions for $n_{s}$ and $r$, which depend on the ratio $\left(\beta_{\tilde{\lambda}} / \tilde{\lambda}\right)_{\star}$ with $\lambda_{\star}$ fixed, depend on the running. Different boundary conditions at the EW scale will result in different values of $\mathcal{M}$ required to adjust the matching conditions at $t_{\mathrm{eq}}$ in such a way that $\tilde{\lambda}_{\star}=4 \cdot 10^{-10}$ is obtained. Furthermore, different values of $\mathcal{M}$ (and $\lambda$ ) at the matching point will give a different value for $\tilde{y}_{\text {eq }}$. Since $\beta_{\tilde{y}_{t}} \approx 0, \tilde{y}_{\mathrm{eq}}=\tilde{y}_{\star}$ this value will determine the correction to the inflationary parameters.

Given the simple form of eq. (5.9) we can integrate $d \tilde{\lambda} / d t=\beta_{\tilde{\lambda}}$ explicitly

$$
\tilde{\lambda}(t)=\tilde{\lambda}_{\mathrm{eq}}+\left(t-t_{\mathrm{eq}}\right) \beta_{\tilde{\lambda}}=\tilde{\lambda}_{\mathrm{eq}}+\ln \left(\frac{\tilde{y} \tilde{\phi}}{y_{\mathrm{eq}} \phi_{\mathrm{eq}}}\right) \beta_{\tilde{\lambda}}
$$

It is possible to express $\tilde{\lambda}$ as a function of the field $h$ and the low energy parameters at the matching point $t_{\text {eq }}$. Using eqs. (5.9) and (5.10) we have

$$
\tilde{\lambda}\left(h, t_{\mathrm{eq}}\right)=6^{4 / 3} \lambda_{\mathrm{eq}}^{1 / 3} \mathcal{M}^{8 / 3}+\beta_{\tilde{\lambda}}\left(t_{\mathrm{eq}}\right) \ln \left(\frac{h^{1 / 3} y_{\mathrm{eq}} \lambda_{\mathrm{eq}}^{-1 / 6} \mathcal{M}^{2 / 3} 6^{1 / 3}}{m_{t}^{\mathrm{EW}} e^{t_{\mathrm{eq}}}}\right),
$$

where we used $y_{\mathrm{eq}} \phi_{\mathrm{eq}}=m_{t}^{\mathrm{EW}} e^{t_{\mathrm{eq}}}$ and (5.3). Once we determine $t_{\mathrm{eq}}$, the corrections to $n_{s}$ and $r$ proportional to $\beta_{\tilde{\lambda}}\left(t_{\text {eq }}\right) / \lambda_{\star}$ can be computed. The value of eq. (5.12) at the field value $h_{\star}$ is fixed by eq. $(5.8)$, i.e. $\tilde{\lambda}\left(h_{\star}, t_{\mathrm{eq}}\right)=\lambda_{\star}$. This, together with the relation defining the boundary eq. (5.7) forms a system of two equations with four unknowns $\left\{t_{\text {eq }}, h_{\star}, \mathcal{M}, h_{\text {end }}\right\}$. In order to close the system we add the equation for the number of $e$-folds (if not otherwise specified we use $\left.N_{\star}=60\right)$ and $\epsilon_{V}\left(h_{\text {end }}\right)=1$. Summarizing, we want to solve the following system of equations

$$
\tilde{\lambda}\left(h_{\star}, t_{\mathrm{eq}}\right)=\tilde{\lambda}_{\star}, \quad \mathcal{M}=\frac{\lambda_{\mathrm{eq}}^{1 / 4}}{\sqrt{2} y_{\mathrm{eq}}} m_{t}^{\mathrm{EW}} e^{t_{\mathrm{eq}}}, \quad N_{\star}=\int_{h_{\mathrm{end}}}^{h_{\star}} \frac{d h}{\sqrt{2 \epsilon}}, \quad \epsilon_{V}\left(h_{\mathrm{end}}\right)=1 .
$$

We used $\phi_{\text {eq }}=y_{\text {eq }}^{-1} m_{t}^{\mathrm{EW}} e^{t_{\mathrm{eq}}}$ to rewrite eq. (5.7) in terms of $\mathcal{M}$ in the second equation. In practice we do not solve explicitly the last equation but approximate $h_{\star} \gg h_{\text {end }} \simeq 0$ as it turns out that $h_{\text {end }}$ is always one or two orders of magnitude smaller than $h_{\star}$. The values for $\left\{\tilde{\lambda}_{\star}, N_{\star}, m_{t}^{\mathrm{EW}}\right\}$ are fixed. Further, $\lambda_{\text {eq }}, y_{\text {eq }}$ are the SM running couplings evaluated at $t_{\text {eq }}$; they depend implicitly on the boundary conditions at the electroweak scale for $\lambda, y$, i.e. on the mass of the top and the Higgs measured at the LHC.

\subsubsection{Boundary conditions at the electroweak scale}

First we discuss the results without threshold corrections, when the observables only depend on the boundary conditions at the EW scale. Without running, $\mathcal{M}$ would be fixed by $\tilde{\lambda}_{\star}$ in eq. (5.8) via eq. (3.1), and its constant value would define the matching point 
eq. (5.7). Instead $\left\{\mathcal{M}, t_{\mathrm{eq}}\right\}$ are coupled by the first two equations in (5.13). Before solving it numerically, it is useful to build some idea about what results to expect. For this we solve $\mathcal{M}$ from the first equation by neglecting the running of $\tilde{\lambda}$ in the large field regime, i.e. the second term in eq. (5.12); this will give some numerical correction but does not change the qualitative nature of the solution. We find $\sqrt{6} \mathcal{M} \simeq \tilde{\lambda}_{\star}^{3 / 8} \lambda_{\text {eq }}^{-1 / 8}$. Substituting in the second equation, and solving for $\lambda_{\text {eq }}$ gives

$$
\lambda_{\mathrm{eq}}=\lambda_{\star}\left(\frac{y_{\mathrm{eq}}}{\sqrt{3} m_{t}^{\mathrm{EW}} e^{t_{\mathrm{eq}}}}\right)^{8 / 3} e^{-8 t_{\mathrm{eq}} / 3} \equiv \alpha e^{-8 t_{\mathrm{eq}} / 3} .
$$

Thus $\lambda_{\text {eq }}$ is given by the intersection of the two curves

$$
\lambda(t)=\alpha e^{-8 t / 3},
$$

with $\alpha$ depending (weakly) on the boundary conditions at the EW scale. For example, for fixed Higgs mass a larger top mass will give a larger matching point $t_{\text {eq }}$, and thus a smaller $\lambda_{\text {eq }}$ as the coupling value decreases with renormalization time $t$ (see figure $2 \mathrm{~b}$ ). To first approximation we have that the corrections to the inflationary parameters go as $\left.\left(\beta_{\tilde{\lambda}} / 4 \tilde{\lambda}\right)\right|_{\star}$. From eq. (5.9) and eq. (5.12) we arrive at

$$
\left.\left(\frac{\beta_{\tilde{\lambda}}}{4 \tilde{\lambda}}\right)\right|_{\star}=\left.\left(\frac{\beta_{\tilde{\lambda}}}{4 \tilde{\lambda}}\right)\right|_{\text {eq }}\left(1+\left.\left(\frac{\beta_{\tilde{\lambda}}}{4 \tilde{\lambda}}\right)\right|_{\text {eq }} \ln (\ldots)\right)^{-1},\left.\quad\left(\frac{\beta_{\tilde{\lambda}}}{4 \tilde{\lambda}}\right)\right|_{\text {eq }}=-\frac{3 y_{\text {eq }}^{4}}{32 \pi^{2}} \frac{1}{\lambda_{\text {eq }}} \propto \frac{1}{\lambda_{\text {eq }}} .
$$

It follows that the corrections to the observables parameterized by $\left|\beta_{\tilde{\lambda}} /(4 \tilde{\lambda})\right|_{\star}$ increase for smaller $\lambda_{\text {eq }}$, i.e. for a larger top mass. This is illustrated in the left plot of figure $2 \mathrm{~b}$. In eq. (5.16) $\ln (\ldots)$ is the $\log$ appearing in eq. (5.12), which numerically is order $\mathcal{O}(10)$ for different boundary conditions. The log enhances the size of the corrections as is shown in the left plot of figure 3 . For example, for boundary conditions such that $\beta_{\tilde{\lambda}} /\left.(4 \tilde{\lambda})\right|_{\text {eq }}=10^{-2}$ $10^{-1}$, the corrections are already order one. For larger corrections it is no longer possible to obtain $N_{\star}=60 e$-folds of inflation. This can also be seen from figure 2a: the larger the top mass the larger the correction, but we see that this also pushes the maximum of the potential to smaller Higgs field values until the region on the left is too small to support $60 e$-folds.

The full numerical results for the observables $n_{s}$ and $r$ are shown in figure 4a for different top masses. We also plotted the contours of the 2015 and 2018 Planck data [1, 62]. Although the tree-level results are outside the $2 \sigma$-contours of the latest Planck data, the running corrections can bring the model back into the region favored by Planck.

\subsubsection{Threshold corrections}

Let us now include threshold corrections, which we model by a shift in $\lambda \rightarrow \lambda+\Delta \lambda$ at the boundary between the small and large field regime. $\Delta \lambda$ has to be considered as the sum of the contributions from higher dimensional operators to the running of $\lambda[5,6,11-15]$. This means that at $t_{\text {eq }}$, the value of $\lambda$ that is matched to the tilde parameters is shifted by

$$
\lambda_{\text {eq }}^{\text {new }}=\lambda\left(t_{\text {eq }}\right)+\Delta \lambda
$$


Case A

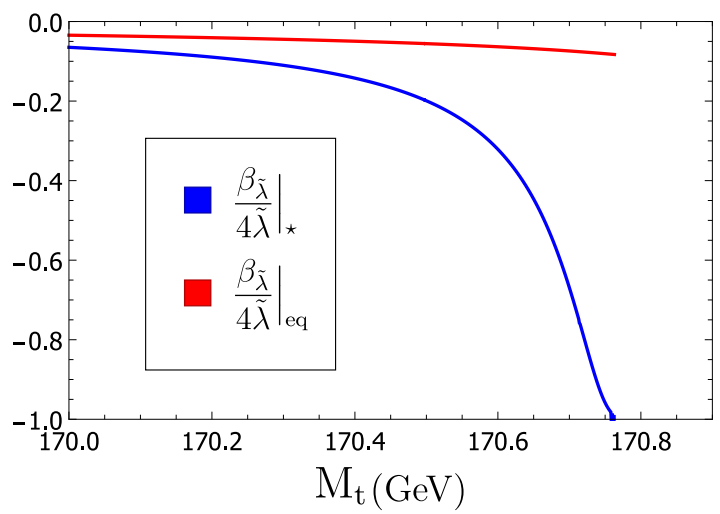

Case B

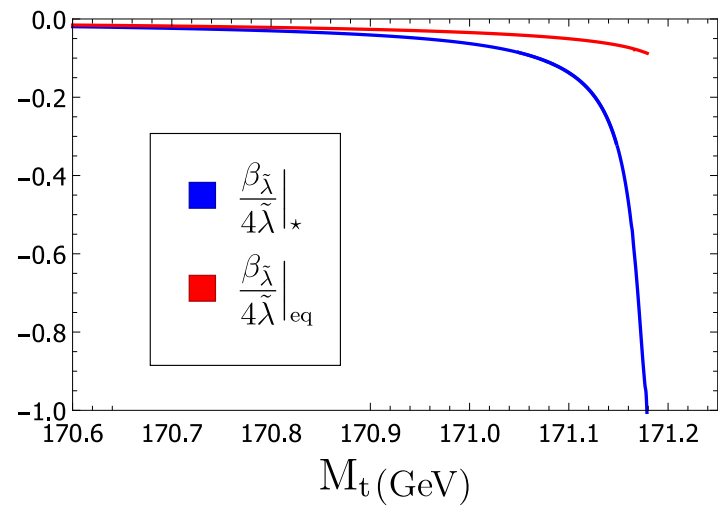

Figure 3. The value of $\left(\beta_{\tilde{\lambda}} / \tilde{\lambda}\right)_{\text {eq }}$ (red curves) depends only on the SM couplings at the EW scale and on the running (no threshold corrections are included). Its value translates in the actual size of the RG correction (blue curves) through the enhancement given in eq. (5.16). For $\left(\beta_{\tilde{\lambda}} / \tilde{\lambda}\right)_{\star}<-1$, the potential cannot sustain $60 e$-folds of inflation anymore. In case $\mathrm{B}$, due to the gauge boson contributions, the absolute value of the correction takes almost a step function shape. This causes a smaller region in parameter space with respect to case A for which the predictions are sensitive to the RG flow.

If we assume as in [63] that new physics implies a shift in the beta function of $\lambda$ on the order of $\delta \beta_{\lambda} \sim 1 /(4 \pi)^{2}$ at the matching scale, the effective shift seen at the inflationary scale by $\lambda$ would be given approximately by $\Delta \lambda \sim \delta \beta_{\lambda} \ln \left(\phi_{*} / \phi_{\mathrm{eq}}\right) \sim 10^{-2}$. In principle there is also a jump in the Yukawa coupling, which we ignore (this gives a degeneracy with the EW boundary value of the Yukawa coupling) as the relative correction is small. To see the effect of the threshold corrections, we solve the same system of equations (5.13), but with the substitution $\lambda_{\mathrm{eq}} \rightarrow \lambda_{\mathrm{eq}}^{\text {new }}$. We fix the boundary conditions at the EW scale and let $\Delta \lambda$ vary. In order to understand the numerical results we can go through the same steps as before, with the only difference that (5.15) now becomes $\lambda(t)+\Delta \lambda=\alpha e^{-8 t / 3}$. For fixed EW boundary conditions $t_{\text {eq }}$ is now given by the intersection between the shifted curve and the same $\alpha e^{-8 t / 3}$ as before. It follows that a positive/negative $\Delta \lambda$ will cause a smaller/larger correction that goes "up/down" in the $\left(n_{s}, r\right)$-plot compared to the tree level result, as illustrated in figure $2 \mathrm{~b}$.

The predictions for $n_{s}$ and $r$ in presence of threshold correction are shown in figure $4 \mathrm{~b}$, where we now also showed the band range for $N_{\star}=50-60$. The curve trajectory in the $\left(n_{s}, r\right)$-plot obtained by decreasing the shift $\Delta \lambda$ is fully degenerate with the curve obtained by increasing the top mass, as can be seen by comparing with figure $4 \mathrm{a}$. This can easily be understood by looking at the systems of equations solved, and can also be clearly seen from figure $2 \mathrm{~b}$ : increasing the top mass and decreasing the shift $\Delta \lambda$ both have the same effect of decreasing $\lambda_{\text {eq }}$, and thus increasing the RG corrections to inflation.

\subsection{Matching and running: other cases}

In case B the gauge bosons are back in the spectrum during inflation. We proceed in the same way as in the previous section, but with different RGEs in the large field regime eq. (4.17) with $\left(f_{1}, f_{2}\right)=(1,1)$. The running of $\tilde{\lambda}$ depends on the gauge couplings only 

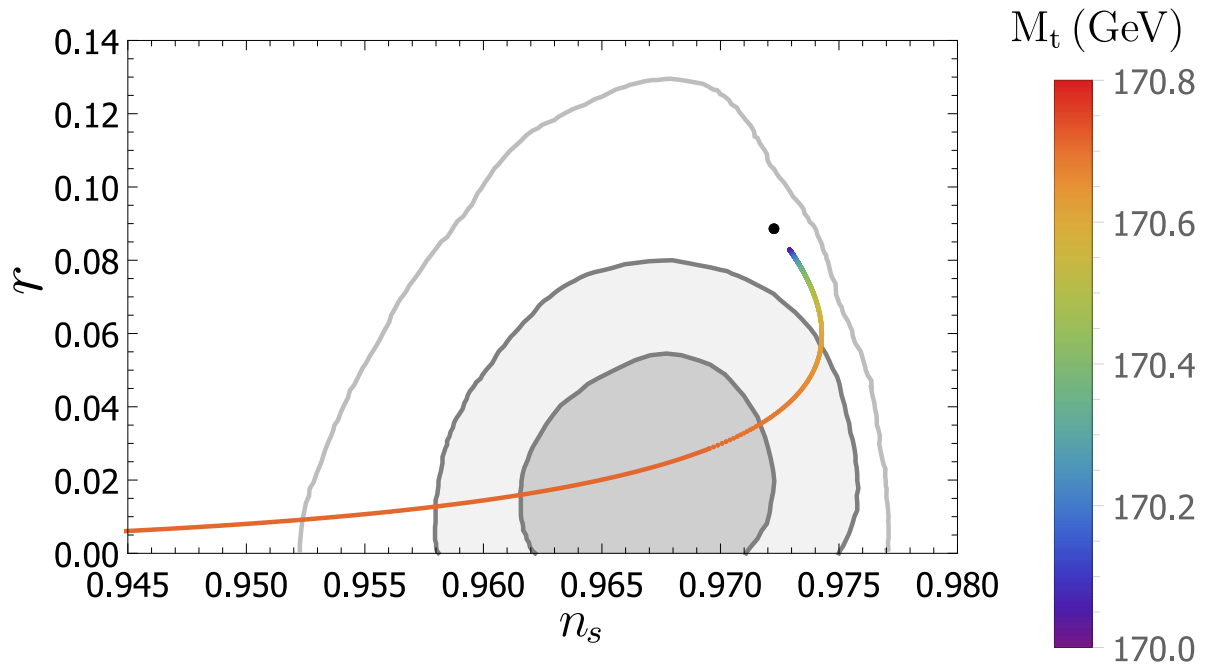

(a) Inflationary predictions for varying top mass. The black dot represents the tree level result that is reached for unrealistic values $M_{\mathrm{t}}<160 \mathrm{GeV}$.

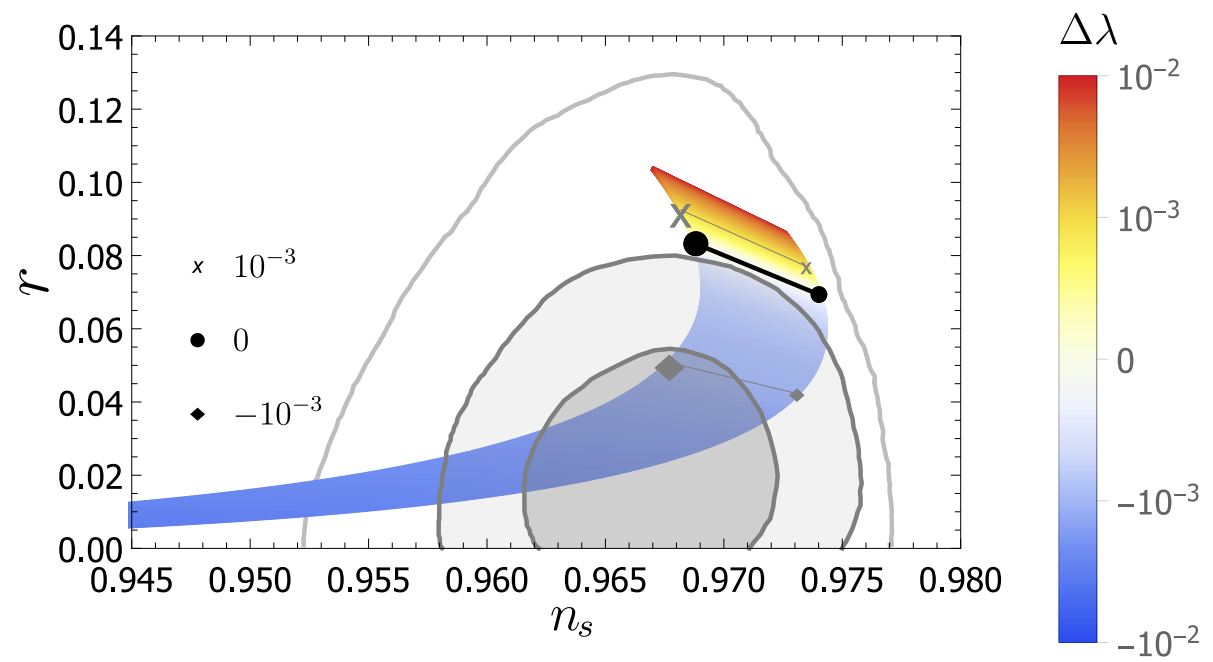

(b) Inflationary predictions for $M_{t}=170.5 \mathrm{GeV}$ and $M_{h}=125.6 \mathrm{GeV}$ fixed and varying threshold conditions. The cross, circle, and diamand markers correspond to $\Delta \lambda=10^{-3}, 0$ and $10^{3}$ respectively. Results are shown for the range $N_{*}=50$ (big marker) to $N_{*}=60$ (small marker). Note that, in agreement with figure $2 \mathrm{~b}$, negative kicks lead to a bigger spread in the predictions.

Figure 4. Case A: predictions for the spectral index $n_{s}$ and tensor-to-scalar ratio $r$ compared to the 2018 ( 1 and $2 \sigma$ in the grey shaded regions) and the 2015 Planck data (the external light grey line is the $2 \sigma$ contour). The effect of threshold corrections is completely degenerate with changing EW boundary conditions.

through the combinations $g_{i}^{2} / \tilde{\alpha_{0}}$, and thus we add to eq. (5.10) the following matching condition for the gauge couplings

$$
\tilde{g}_{i \mathrm{eq}}^{2} \equiv \frac{g_{\mathrm{ieq}}^{2}}{\tilde{\alpha}_{0 \mathrm{eq}}}=g_{i \mathrm{eq}}^{2} \lambda_{\mathrm{eq}}^{-1 / 3} \alpha_{0}^{-1} \mathcal{M}^{4 / 3}
$$

The results of the numerical implementation for case B (without threshold corrections) are given in figure 5. As in case A the tree level results are never reached for realistic 

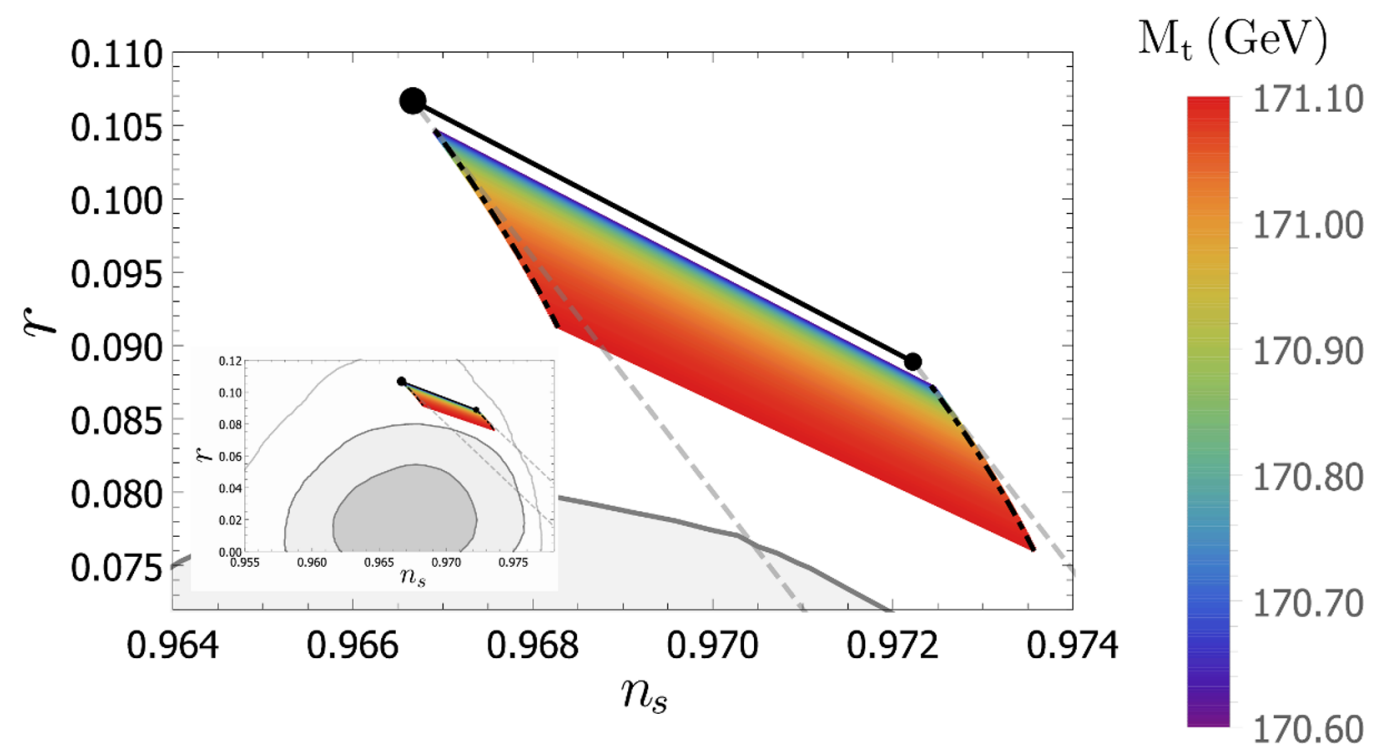

Figure 5. $\left(n_{s}, r\right)$ predictions for Case B without threshold corrections and for $\alpha_{0}=1$. The dashed line represents the analytic approximation of eq. (5.6). We have zoomed in on the Planck plot since the inflationary observables are less RG dependent than in case A. This outcome can be explained (see main text) by looking at figure 3 .

values of the top mass. However, for the values of the boundary conditions that allow inflation to happen, there is less dependence on the RG flow. This can be explained by looking at the right-hand plot in figure 3 . The positive contribution from the gauge bosons to $\beta_{\tilde{\lambda}}$ leads to $\left(\beta_{\tilde{\lambda}} / \tilde{\lambda}\right)_{\text {eq }}$ changing rapidly over a relatively narrow range of top masses of $\mathcal{O}(0.1) \mathrm{GeV}$, roughly between $171-171.2 \mathrm{GeV}$. Since the corrections proportional to $\left(\beta_{\lambda} / \lambda\right)_{\star}$ are enhanced through eq. (5.16), we jump quickly from having zero corrections to spoiling inflation completely, i.e. from $\left(\beta_{\lambda} / \lambda\right)_{\star} \simeq 0$ to $\left(\beta_{\lambda} / \lambda\right)_{\star} \simeq-1$. This also means that the inflationary predictions cannot be brought back in the $2 \sigma$-contour of the latest Planck data by the RG flow corrections. Since changing EW boundary conditions and varying matching corrections is degenerate, this result is robust.

This is in contrast with case A, as can be seen from the left-hand plot in figure 3 . In this case the corrections grow much more gradually with changing top mass, and change from percent level to order one over a much wider range of top masses of $\mathcal{O}(1) \mathrm{GeV}$. As a result the inflationary predictions vary over a wider range as well, and as noted before, in case $\mathrm{A}$ it is possible that the RG corrections bring the predictions back in the region favored by Planck.

In case $\mathrm{C}$ the implementation is very similar to the previous cases with the quantitative difference that now, since the top quark decouples, $\beta_{\tilde{\lambda}}>0$ in the large field regime. This implies positive corrections to $n_{s}$ and $r$, as can be understood from the approximate solution eq. (5.6). Thus, by increasing the top mass (i.e. increasing the size of the corrections), the inflationary parameters "move up" in the Planck plot to higher values of the tensor-toscalar ratio $r$ compared to the tree-level result, farther outside region favored by Planck. Finally, case D gives trivially the tree level results since $\beta_{\tilde{\lambda}}=\mathcal{O}\left(\delta^{-1}\right)$. 


\section{Conclusions}

We studied the effects of the renormalization group flow on inflationary models that are embedded in low energy (beyond the) Standard Model theories. The inflationary predictions depend on the renormalization group equations during the inflationary stage and on a new kind of UV sensitivity [5] present in inflationary models with non-renormalizable operators.

In this paper we developed the necessary tools to analyse the effects of the running couplings, and of the threshold corrections parameterizing the UV physics, on the inflationary predicitons. Results have been applied to the SM with non-derivative couplings to gravity and different cases have been considered in which apart from the Higgs also gauge boson/fermions are non-minimally coupled to gravity.

We have calculated the one-loop beta functions in the large field regime using a covariant approach that takes into account the non-trivial geometry of the field space manifold. The results for the RGEs are summarized in eq. (4.17). Given the simple form of the beta functions, it may seem that by introducing the covariant formalism we used a sledgehammer to crack a nut. However, as we saw for example in case B, the covariant formalism is necessary to have a consistency check and thus obtain reliable results. This suggests that in general a covariant formalism is desirable to compute the RGEs in presence of a non-flat metric in field space.

The explicit dependence of $n_{s}$ and $r$ on the running coupling $\tilde{\lambda}$ defined in eq. (3.1) can be computed via an analytical approximation with results given in eq. (5.6). In inflationary models in which, in the large field limit, the potential asymptotes to a constant value exponentially fast as a function of the canonical field, the RG corrections to $n_{s}$ and $r$ disappear at first order in the $1 / N_{\star}$ expansion due to a cancellation between the running dependence of the slow-roll parameters and of the number of $e$-folds. This is the case for the large class of cosmological attractors that includes Higgs inflation as a particular case $[6,29]$. As eq. (5.6) shows, such an insensitivity to running effects does not happen in general, and not in models where the approach to a constant is polynomial.

The full non-linear RG dependence for the original new Higgs inflation proposal with only a non-minimal coupling for the Higgs field (case A) is shown in figure 4a (as a function of the boundary conditions at the EW scale), and in figure $4 \mathrm{~b}$ (as a function of the threshold corrections). In figure 5 the sensitivity of the predictions when the gauge bosons are also non-minimally coupled (case B) is illustrated. In both cases the tree level results are modified by the running corrections. In case A this allows to 'push back' the prediction into the region favored by the Planck data, whereas in case B the running corrections ruin the flatness of the inflationary potential before the $2 \sigma$-region is reached.

The general lesson is that in these type of models the classical description is not enough to sensibly compare the predictions to the Planck data.

\section{Acknowledgments}

We thank Cristiano Germani and Sander Mooij for helpful discussions. JF is supported by the European Research Council under the European Union's Horizon 2020 research and 
innovation programme (grant agreement No. 758792, project GEODESI). When this work started MP and JF were supported by the Projectruimte "Keeping track of time during inflation" grant from the Dutch Organization for Scientific Research (NWO).

\section{A Calculation of the self-energies}

In this appendix we provide more details for the calculation of the self-energies of the Higgs, Goldstone, fermion and gauge fields. We first determine the self-energies in the AbelianHiggs model augmented with a single fermion. This not only simplifies the calculation of the two-point funtions but also avoids much notational clutter. Sections A.1 to A.3 give the expressions for the $\mathrm{U}(1)$ theory. In appendix A.4 we calculate the two-point funtions for the Higgs, gauge and fermion field in the Abelian model. These results for the self-energies are subsequently generalized to the full SM gauge group in appendix A.5.

\section{A.1 Renormalized action}

We give the Lagrangian, counterterms and Feynman rules for the Abelian-Higgs model with a single Dirac fermion field. Rescaling the bare parameters eq. (3.3) the renormalized action is

$$
\begin{aligned}
\mathcal{L}= & -\frac{1}{2} K_{\phi}\left(\varphi^{a}\right) Z_{\phi} \partial_{\mu} \varphi^{a} \partial^{\mu} \varphi_{a}-\frac{1}{4} K_{A}\left(\varphi^{a}\right) Z_{A}\left(F_{\mu \nu}\right)^{2}+K_{\psi}\left(\varphi^{a}\right) Z_{\psi} \bar{\psi}(i \not \partial) \psi \\
& -Z_{\phi}^{2} Z_{\lambda} V\left(\varphi^{a}\right)-Z_{\psi} Z_{y} Z_{\phi}^{1 / 2} \frac{y}{\sqrt{2}} \bar{\psi} F_{\psi}\left(\varphi^{a}\right) \psi-Z^{2} Z_{A} Z_{\phi} \frac{1}{2} g^{2} A^{2} F_{A}\left(\varphi^{a}\right) \\
& +Z_{g} Z_{A}^{1 / 2} Z_{\psi}\left(g q_{L} \bar{\psi} A P_{L} \psi+g q_{R} \bar{\psi} A P_{R} \psi\right)+Z_{g} Z_{A}^{1 / 2} Z_{\phi}^{1 / 2} K_{\phi}\left(\varphi^{a}\right) g q_{H} A(\varphi \partial \theta-\theta \partial \varphi) .
\end{aligned}
$$

With the charge of the Higgs field fixed to unity, gauge invariance implies the relation $q_{L}-q_{R}+q_{H}$ for the charges of the left and right handed fermions and the Higgs field. We can then identify the U(1) symmetry with hypercharge by equating $2 Y_{i}=q_{i}$. Further,

$$
\begin{aligned}
& V=\frac{\lambda}{4}\left(\varphi^{2}+\theta^{2}\right)^{2}, \quad F_{\psi}=\left(\varphi-i \gamma^{5} \theta\right), \quad F_{A}=K_{\phi}\left(\varphi^{2}+\theta^{2}\right), \\
& K_{\phi}=\left(1+Z_{\delta} \delta\right), \quad K_{\psi}=\left(1+Z_{\alpha_{F}} Z_{\delta} \alpha_{F} \delta\right), \quad K_{A}=\left(1+Z_{\alpha_{A}} Z_{\delta} \alpha_{A} \delta\right)
\end{aligned}
$$

with $\delta$ given in (2.4), and we introduced the notation $Z_{\delta}=Z_{\phi}^{2} Z_{\lambda} / Z_{\mathcal{M}}^{4}$. The gauge fixing term for the generalized $R_{\xi}$-gauge is

$$
\mathcal{L}_{\mathrm{GF}}=-\frac{1}{2 \xi} K_{A}(\phi)\left(Z_{A}^{1 / 2} \partial^{\mu} A_{\mu}-Z_{g} Z_{\phi} g \xi \frac{K_{\phi}(\phi) \phi}{K_{A}(\phi)} \theta\right)^{2}
$$

The $A_{\mu} \partial^{\mu} \theta$-term cancels the interaction in the Higgs kinetic terms, and the quadratic terms are diagonal. We work in Landau gauge $\xi=0$, for which the ghosts fields decouple. 


\section{A.2 Feynman rules}

In this subsection we will give the Feynman rules for the action in terms of the covariant fields. We expand the action eqs. (A.1) and (A.3) in covariant fields using eqs. (3.9) and (3.10), where we use the notation in eq. (3.8). This gives the interaction Lagrangian which the defines the various couplings

$$
\begin{aligned}
& \mathcal{L}_{\text {int }}=-\lambda_{m Q^{\phi} n Q^{\theta}}\left(Q^{\phi}\right)^{m}\left(Q^{\theta}\right)^{n}-y_{m Q^{\phi} n Q^{\theta}}\left(Q^{\phi}\right)^{m}\left(Q^{\theta}\right)^{n} \bar{\psi}\left(i \gamma^{5}\right)^{n} \psi \\
& -y_{2 \psi} \bar{\psi} \psi+g_{L} \bar{\psi} Q^{A} P_{L} \psi+g_{R} \bar{\psi} Q^{\phi} P_{R} \psi
\end{aligned}
$$

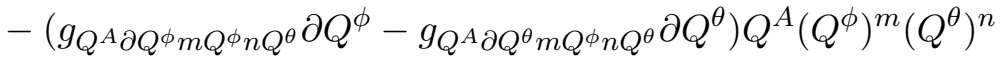

$$
\begin{aligned}
& -g_{2 Q^{A} m Q^{\phi} n Q^{\theta}}\left(Q^{A}\right)^{2}\left(Q^{\phi}\right)^{m}\left(Q^{\theta}\right)^{n}+\ldots
\end{aligned}
$$

Equivalently we expand the kinetic terms, for example

$$
\mathcal{L}_{\mathrm{k}}=-\mathcal{K}_{Q^{\phi} 2 \partial Q^{I}}\left(Q^{\phi}\right)^{2}\left(\partial Q^{I}\right)^{2}-\mathcal{K}_{2 Q^{\theta} 2 \partial Q^{I}}\left(Q^{\theta}\right)^{2}\left(\partial Q^{I}\right)^{2}+\ldots
$$

All interactions are defined with a minus sign (the only exception is for one of the derivative interactions and the fermion-gauge interaction), and without numerical factors. This means that for a vertex with $m Q^{\phi}$-fields and $n Q^{\theta}$-fields and with or without fermion/gauge lines we have, respectively:

$$
\begin{aligned}
V^{\left(m Q^{\phi} n Q^{\theta}\right)} & =(-i) m ! n ! \lambda_{m Q^{\phi} n Q^{\theta},} \\
V^{\left(m Q^{\phi} n Q^{\theta} 2 \psi\right)} & =(-i) m ! n ! y_{m Q^{\phi} n Q^{\theta}}\left(i \gamma^{5}\right)^{n}, \\
V^{\left(m Q^{\phi} n Q^{\theta} 2 Q^{A}\right)} & =(-i) 2 ! m ! n ! g_{2 Q^{A} m Q^{\phi} n Q^{\theta} .}
\end{aligned}
$$

For the derivative interaction we get

$$
\begin{aligned}
V^{\left(Q^{A} \partial Q^{j} m Q^{\phi} n Q^{\theta}\right)} & =-i g_{Q^{A} \partial Q^{j} m Q^{\phi} n Q^{\theta}}\left(-i p^{\mu}\right), & & j=\{\phi, \theta\} \\
V^{2 Q^{j} 2 \partial Q^{I}} & =2 ! 2 !\left(-i \mathcal{K}_{\left.2 Q^{j} 2 \partial Q^{I}\right) p^{\mu} p_{\mu},}\right. & & j=\{\phi, \theta\}
\end{aligned}
$$

with $p$ the momentum running through the vertex. The fermion, scalar and gauge propagators are given by:

$$
\begin{aligned}
&-i D_{\psi}(p)=K_{\psi}^{-1} \frac{-i\left(-\not p+m_{\psi}\right)}{p^{2}+m_{\psi}^{2}-i \epsilon} \\
&-i D_{Q^{I}}(p)=K_{\phi}^{-1} \frac{-i}{p^{2}+\left(m^{2}\right)_{I}^{I}-i \epsilon} \\
&-i D_{\mu \nu}(p) \stackrel{\xi_{G}=0}{=}-i K_{A}^{-1} \frac{g_{\mu \nu}-\frac{p_{\mu} p_{\nu}}{p^{2}}}{p^{2}+m_{A}^{2}-i \epsilon}
\end{aligned}
$$

The masses are given in eqs. (4.2) and (4.3).

Finally, we need the countertems in the quadratic action, We define the notation

$$
\mathcal{L}_{\mathrm{ct}}=-\sum_{I}\left(\frac{1}{2} Z_{2 Q^{I}}\left(\partial Q^{I}\right)^{2}+Z_{c_{I} 2 Q^{I}} c_{c_{I} 2 Q^{I}}\left(Q^{I}\right)^{2}\right)+Z_{2 \psi_{L, R}} \bar{\psi}_{L, R} \not \partial \psi_{L, R}-Z_{y 2 \psi} y_{2 \psi} \bar{\psi} \psi
$$


with $I$ running over the bosonic fields and $c_{I}=\{\lambda, g\}$ labels the coupling that the counterterm normalizes. The counterterm can be expressed in terms of the "elementary" ones eq. (3.3) as

$$
\begin{aligned}
Z_{2 Q^{\phi}} & =Z_{2 Q^{\theta}}=Z_{\lambda} Z_{\phi}^{3} Z_{\mathcal{M}}^{-4}, & Z_{\lambda 2 Q^{\phi}} & =Z_{\lambda 2 Q^{\theta}}=Z_{\lambda} Z_{\phi}^{2}, \\
Z_{2 Q^{A}} & =Z_{A} Z_{K_{A}}, & Z_{g 2 Q^{A}} & =Z_{K_{A}} Z_{g}^{2} Z_{A} Z_{\alpha_{A}}^{-1}, \\
Z_{2 \psi_{L, R}} & =Z_{\psi_{L, R}} Z_{K_{\psi}}, & Z_{y 2 \psi} & =Z_{\psi} Z_{y} Z_{\phi}^{1 / 2},
\end{aligned}
$$

with

$$
Z_{K_{A}}=Z_{\alpha_{0 A}}\left(Z_{\lambda} Z_{\phi}^{2} Z_{\mathcal{M}}^{-4}\right)^{1+\frac{n_{A}}{2}}, \quad Z_{K_{\psi}}=Z_{\alpha_{0 f}}\left(Z_{\lambda} Z_{\phi}^{2} Z_{\mathcal{M}}^{-4}\right)^{1+\frac{n_{f}}{2}}
$$

\section{A.3 Coupling strengths}

We list the relevant vertices in the theory with a $\mathrm{U}(1)$ gauge group for case $\mathrm{B}$, where both fermions and gauge bosons are in the spectrum. Case A can be obtained from this result by setting $g \rightarrow g K_{A}(\phi)$, and then subsequently integrating out the gauge bosons; thus effectively we can set the gauge coupling to zero. Case $\mathrm{C}$ is obtained by rescaling the Yukawa coupling $y \rightarrow y / K_{\psi}$, which makes the fermion contribution subleading, and effectively we can set the Yukawa to zero. Case D is obtained by rescaling both $g$ and $y$ with the appropriate metric factors; the net effect is that both fields decouple and we can effectively set both couplings to zero. All couplings are evaluated on the background and we use the notation that the first term in the curly brackets is the leading result in the SM limit $(\delta \ll 1)$, and the second term the leading result in the inflationary regime $(\delta \gg 1)$.

The vertices derived from the potential are valid in all four cases. For example, $4 ! \lambda_{4 Q^{\phi}}=\nabla_{\phi}^{4} V$ and $3 ! \lambda_{Q^{\phi} 2 Q^{\theta}}=\nabla_{(\theta} \nabla_{\theta} \nabla_{\phi)} V$. The full results are given by

$$
\begin{aligned}
& \lambda_{2 Q^{\phi}}=\frac{\lambda \phi^{2}}{2}\{3,1\}, \quad \lambda_{2 Q^{\theta}}=\frac{\lambda \phi^{2}}{2}\{1,3\}, \quad \lambda_{3 Q^{\phi}}=\frac{\lambda \phi}{3}\{3,-1\}, \quad \lambda_{4 Q^{\phi}}=\frac{\lambda}{12}\{3,5\}, \\
& \lambda_{4 Q^{\theta}}=\frac{\lambda}{4}\{1,-9\}, \quad \lambda_{Q^{\phi} 2 Q^{\theta}}=\lambda \phi\{1,-3\}, \quad \lambda_{2 Q^{\phi} 2 Q^{\theta}}=\frac{\lambda}{2}\{1,15\} .
\end{aligned}
$$

The vertices derived from the Yukawa interactions are $y_{Q^{\phi}}=\nabla_{\phi} F_{\psi}, y_{Q^{\theta}}=i \gamma_{5} \nabla_{\theta} F_{\psi}$ etc.

$$
y_{Q^{\phi}}=\frac{y}{\sqrt{2}}\{1,1\}, \quad y_{Q^{\theta}}=\frac{y}{\sqrt{2}}\{1,1\}, \quad y_{2 Q^{\phi}}=-\frac{y}{\sqrt{2} \phi}\{0,1\}, \quad y_{2 Q^{\theta}}=-\frac{y}{\sqrt{2} \phi}\{0,1\} .
$$

The vertices involving gauge fields are

$$
\begin{aligned}
g_{2 Q^{A} 2 Q^{\phi}} & =\frac{g^{2}}{6}\{3,-5 \delta\}, & g_{2 Q^{A} 2 Q^{\phi}} & =\frac{g^{2}}{2}\{1,5 \delta\}, \\
g_{Q^{A} \partial Q^{\phi} Q^{\theta}} & =g\{1, \delta\}, & g_{A \bar{\psi}_{s} \psi_{s}} & =g q_{s}\{1,1\} .
\end{aligned}
$$

with $s=L, R$. The relevant couplings coming from the kinetic terms are

$$
\mathcal{K}_{2 \partial Q^{A} 2 Q^{\phi}}=\frac{\alpha_{A 0} \delta}{3 \phi^{2}}\{0,-1\}, \quad \mathcal{K}_{2 \partial Q^{A} 2 Q^{\theta}}=\frac{\alpha_{A 0} \delta}{\phi^{2}}\{0,1\} .
$$




\section{A.4 Two-point functions in the Abelian-Higgs model}

With the Feynman rules in hand we can calculate the one-loop self-energies of the Higgs, Goldstone, fermion, and U(1) gauge boson field. We only include the interactions from the kinetic terms eq. (A.14) that are not suppressed by $\delta^{-1}$. The results are

$$
\begin{aligned}
& \Pi_{Q^{\phi}}\left(p^{2}\right)=\frac{1}{8 \pi^{2} \epsilon}\left\{\left(18 \lambda_{3 Q^{\phi}}^{2} K_{\phi}^{-1}+12 \lambda_{4 Q^{\phi}} m_{h}^{2}\right) K_{\phi}^{-1}+\left(2 \lambda_{Q^{\phi} 2 Q^{\theta}}^{2} K_{\theta}^{-1}+2 \lambda_{2 Q^{\phi} 2 Q^{\theta}} m_{\theta}^{2}\right) K_{\theta}^{-1}\right. \\
& +\left(6 g_{2 Q^{A} 2 Q^{\phi}} m_{A}^{2}+6 g_{2 Q^{A} Q^{\phi}}^{2}+\frac{3}{4} K_{\theta}^{-1}\left(g_{Q^{A} \partial Q^{\phi} Q^{\theta}}+g_{Q^{A} \partial Q^{\theta} Q^{\phi}}\right)^{2} p^{2}-6 \mathcal{K}_{2 Q^{\phi} 2 \partial Q^{A}} m_{A}^{4}\right) K_{A}^{-1} \\
& \left.-\left(2 y_{Q^{\phi}}^{2}\left(p^{2}+6 m_{\psi}^{2}\right) K_{\psi}^{-1}+8 y_{2 Q^{\phi}} m_{\psi}^{3}\right) K_{\psi}^{-1}\right\}-\left[\left(Z_{\partial Q^{\phi}}-1\right) p^{2} K_{\phi}+\left(Z_{\lambda 2 Q^{\phi}}-1\right) 2 \lambda_{2 Q^{\phi}}\right], \\
& \Pi_{Q^{\theta}}\left(p^{2}\right)=\frac{1}{8 \pi^{2} \epsilon}\left\{\left(12 \lambda_{4 Q^{\theta}} m_{\theta}^{2}+2 \lambda_{2 Q^{\phi} 2 Q^{\theta}} m_{h}^{2}+4 K_{\phi}^{-1} \lambda_{Q^{\phi} 2 Q^{\theta}}^{2}\right) K_{\theta}^{-1}\right. \\
& +\left(6 g_{2 Q^{A} 2 Q^{\theta}} m_{A}^{2}+\frac{3}{4} K_{\phi}^{-1}\left(g_{Q^{A} \partial Q^{\phi} Q^{\theta}}+g_{Q^{A} \partial Q^{\theta} Q^{\phi}}\right)^{2} p^{2}-6 \mathcal{K}_{2 Q^{\phi} 2 \partial Q^{A}} m_{A}^{4}\right) K_{A}^{-1} \\
& \left.-\left(2 y_{Q^{\theta}}^{2}\left(p^{2}+2 m_{\psi}^{2}\right) K_{\psi}^{-1}-8 y_{2 Q^{\theta}} m_{\psi}^{3}\right) K_{\psi}^{-1}\right\}-\left[\left(Z_{\partial Q^{\theta}}-1\right) p^{2} K_{\theta}^{-1}+\left(Z_{\lambda 2 Q^{\theta}}-1\right) 2 \lambda_{2 Q^{\theta}}\right] .
\end{aligned}
$$

For the fermions

$$
\begin{aligned}
\Pi_{\psi}(\not p)= & \frac{1}{8 \pi^{2} \epsilon}\left\{\left(y_{Q^{\phi}}^{2} K_{\phi}^{-1}\left(m_{\psi}-\frac{1}{2} \not p\right)-y_{Q^{\theta}}^{2} K_{\theta}^{-1}\left(m_{\psi}+\frac{1}{2} \not p\right)\right) K_{\psi}^{-1}\right. \\
& \left.+y_{2 Q^{\phi}} K_{\phi}^{-1} m_{h}^{2}-y_{2 Q^{\theta}} K_{\theta}^{-1} m_{\theta}^{2}-3 m_{\psi} K_{A}^{-1} g^{2} q_{L} q_{R}\right\} \\
& -\left[\left(Z_{y 2 \psi}-1\right) m_{\psi}+K_{\psi} \not p P_{L}\left(Z_{2 \psi_{L}}-1\right)+K_{\psi} \not p P_{R}\left(Z_{2 \psi_{R}}-1\right)\right] .
\end{aligned}
$$

For the gauge fields

$$
\begin{aligned}
& \Pi_{\mu \nu}^{A}=\frac{1}{8 \pi^{2} \epsilon}\left\{\left(3 K_{\phi}^{-1} K_{A}^{-1} g_{Q^{\phi} 2 Q^{A}}^{2}+2 K_{\phi}^{-1} g_{2 Q^{\phi} 2 Q^{A}} m_{h}^{2}+2 K_{\theta}^{-1} g_{2 Q^{\theta} 2 Q^{A}} m_{\theta}^{2}\right) g_{\mu \nu}\right. \\
& -K_{\phi}^{-1} K_{\theta}^{-1}\left[\frac{1}{4}\left(g_{Q^{A} \partial Q^{\phi} Q^{\theta}}+g_{Q^{A} \partial Q^{\theta} Q^{\phi}}\right)^{2}\left(\frac{p^{2}}{3}+m_{h}^{2}+m_{\theta}^{2}\right) g_{\mu \nu}\right. \\
& \left.-\left(g_{Q^{A} \partial Q^{\phi} Q^{\theta}}^{2}-g_{Q^{A} \partial Q^{\phi} Q^{\theta}} g_{Q^{A} \partial Q^{\theta} Q^{\phi}}+g_{Q^{A} \partial Q^{\theta} Q^{\phi}}^{2}\right) \frac{1}{3} p_{\mu} p_{\nu}\right] \\
& \left.-\left(\frac{2}{3}\left(p^{2} g_{\mu \nu}-p_{\mu} p_{\nu}\right) g^{2}\left(q_{R}^{2}+q_{L}^{2}\right)+2 g^{2}\left(q_{L}-q_{R}\right)^{2} m_{\psi}^{2} g_{\mu \nu}\right) K_{\psi}^{-2}\right\} \\
& -\left(Z_{2 Q^{A}}-1\right) K_{A}^{-1}\left(p^{2} g_{\mu \nu}-p_{\mu} p_{\nu}\right)-\left(Z_{m_{A}^{2}}-1\right) g_{\mu \nu} m_{A}^{2} .
\end{aligned}
$$

\section{A.5 Two-point functions in the SM}

In this subsection we generalize the $\mathrm{U}(1)$ result to the full $\mathrm{SM}$ gauge $\mathrm{SU}(3) \times \mathrm{SU}(2) \times \mathrm{U}(1)$. We only include the effects of the top quark Yukawa, but neglect the smaller Yukawa 
couplings of the other fermions. To set the notation and our conventions, the covariant derivatives are

$$
\begin{aligned}
D_{\mu} \mathcal{H} & =\left(\partial_{\mu}-i g a_{\mu}^{a} \tau^{a}-i Y_{\mathcal{H}} g^{\prime} B_{\mu}\right) \mathcal{H} \\
D_{\mu} Q_{L} & =\left(\partial_{\mu}-i g_{s} f_{\mu}^{a} t^{a}-i g a_{\mu}^{a} \tau^{a}-i Y_{Q} g^{\prime} B_{\mu}\right) Q_{L} \\
D_{\mu} u_{R} & =\left(\partial_{\mu}-i g_{s} f_{\mu}^{a} t^{a}-i Y_{u} g^{\prime} B_{\mu}\right) u_{R}
\end{aligned}
$$

with $\{B, a, f\}$ the $\mathrm{U}(1), \mathrm{SU}(2)$ and $\mathrm{SU}(3)$ gauge fields respectively with corresponding gauge couplings $\left\{g^{\prime}, g, g_{s}\right\}$. The hypercharge assignments are $Y_{\mathcal{H}}=1 / 2, Y_{Q}=1 / 6$ and $Y_{u}=2 / 3$.

Higgs self energy: $\boldsymbol{\Pi}_{\boldsymbol{h}}$. The Higgs kinetic term can be written in the form

$$
\mathcal{L}_{\mathcal{H}} \supset-K_{\phi}\left(D_{\mu} \mathcal{H}\right)^{\dagger}\left(D^{\mu} \mathcal{H}\right)=-K_{\phi}\left[(\partial \delta \phi)^{2}+\sum_{i=1}^{3}\left(\left(\partial \theta_{i}\right)^{2}-2 g_{i} A^{i}\left(\phi \partial \theta_{i}-\theta_{i} \partial \phi\right)+g_{i}^{2} \phi^{2} A_{i}^{2}\right)+\ldots\right] .
$$

The gauge boson mass eigenstates are $A^{i}=\left\{A^{1}, A^{2}, Z, A_{\gamma}\right\}$ with $W^{ \pm}=A^{1} \pm i A^{2}, Z, A_{\gamma}$ the usual $W, Z$ and photon fields, and the gauge couplings are $g_{i}=\frac{1}{2} \times\left\{g, g, \sqrt{g^{2}+g^{\prime 2}}, 0\right\}$. The Higgs/Goldstone-gauge couplings for the electroweak mass eigenstates in eq. (A.20) are exactly of the form of three massive (and one massless $\mathrm{U}(1)$ gauge bosons) that have eaten the Goldstone bosons $\theta_{i}$. We can choose a gauge fixing term as in eq. (A.3) but now for each pair of $\left\{A^{i}, \theta^{i}\right\}$. Hence, in all loops containing gauge- Higgs interactions we can use a U(1) model and sum over the three gauge couplings [26]. In addition, in loops with gauge-Higgs or gauge-Goldstone interactions, we sum over the $n_{\mathrm{GB}}=3$ three Goldstone bosons. However, However, in all cases (A-D) the Goldstone fluctuations decouple, and this change will not affect the inflationary RGEs.

The difference in the Higgs-fermion Yukawa coupling compared to the $\mathrm{U}(1)$ case is that the top quark is now an $\mathrm{SU}(3)$ triplet. We have to sum over the dimension of the representation which picks up a color factor $N_{c}=3$.

The SM Higgs self energy thus becomes:

$$
\begin{aligned}
& \Pi_{Q^{\phi}}\left(p^{2}\right)= \\
& \frac{1}{8 \pi^{2} \epsilon}\left\{\left(18 \lambda_{3 Q^{\phi}}^{2} K_{\phi}^{-1}+12 \lambda_{4 Q^{\phi}} m_{h}^{2}\right) K_{\phi}^{-1}+\left(2 \lambda_{Q^{\phi} 2 Q^{\theta}}^{2} K_{\theta}^{-1}+2 \lambda_{2 Q^{\phi} 2 Q^{\theta}} m_{\theta}^{2}\right) n_{\mathrm{GB}} K_{\theta}^{-1}\right. \\
& +\sum_{i}\left(6 g_{2 Q^{A^{i}} 2 Q^{\phi}} m_{A^{i}}^{2}+6 g_{2 Q^{A^{i}} Q^{\phi}}^{2}+\frac{3}{4} K_{\theta}^{-1}\left(g_{Q^{A^{i}} \partial Q^{\phi} Q^{\theta^{i}}}+g_{Q^{A^{i}} \partial Q^{\theta^{i}} Q^{\phi}}\right)^{2} p^{2}-6 \mathcal{K}_{2 Q^{\phi} 2 \partial Q^{A^{i}}} m_{A^{i}}^{4}\right) K_{A}^{-1} \\
& \left.-\left(2 y_{Q^{\phi}}^{2}\left(p^{2}+6 m_{\psi}^{2}\right) K_{\psi}^{-1}+8 y_{2 Q^{\phi}} m_{\psi}^{3}\right) N_{c} K_{\psi}^{-1}\right\}-\left[\left(Z_{\partial Q^{\phi}}-1\right) p^{2} K_{\phi}+\left(Z_{\lambda 2 Q^{\phi}}-1\right) 2 \lambda_{2 Q^{\phi}}\right] .
\end{aligned}
$$

The Goldstone self-energy is generalized similarly.

Top quark self energy: $\Pi_{\psi_{f}}$. Consider first the Higgs/Goldstone contribution. The Yukawa interaction is now of the form

$$
\mathcal{L}_{y}=-y\left(\bar{t}_{L} \phi_{0}^{*} t_{R}-\bar{b}_{L} \phi^{-} t_{R}+\text { h.c. }\right)
$$


with $\phi_{0}=\phi+i \theta_{1}$ and $\phi^{-}=\theta_{2}-i \theta_{3}$. The first term is the same as in the Abelian model which reproduces the first four terms in eq. (A.17). The second term gives an additional contribution to $\bar{\psi} P_{R} \psi$, as it can give loops with $\phi^{-}$and $b_{L}$ in the loop. This gives an additional contribution of the same form as the first line in eq. (A.17), but for $\phi, \theta \rightarrow \theta_{1}, \theta_{2}$ and $m_{t} \rightarrow m_{b}$ - except from the $K_{I}^{-1}$-factors, these terms are the same as in the SM regime. The first two terms on the 2 nd line of eq. (A.17), which only arise in the large field regime, originate from the $\phi^{0}$-coupling and are the same as in the $\mathrm{U}(1)$ model.

For the gauge contribution, the last term on the 2nd line in eq. (A.17), to be proportional to the top mass, the gauge interactions have to be diagonal. We thus include the $Z$, $\gamma$ and the QCD contribution. We replace

$$
g^{2} q_{L} q_{R} \rightarrow \sum_{i=\gamma, Z} q_{L}^{i} q_{R}^{i}+g_{s}^{2} C_{2}\left(N_{c}\right)
$$

where $q_{L, R}^{i}$ is the coupling of the left/right-handed top quark to $A^{i}$ (the gauge couplings are absorbed), and $g_{s}$ is the QCD coupling $C_{2}(N)=\left(N^{2}-1\right) /(2 N)$. Except from the $K_{A}^{-1}$-factor these terms are the same the usual SM results.

Although the coefficients of the various terms gets modified in the full SM case, the order in $\delta$ of the various terms remains the same. Thus also in the full SM we find for the current set-up that $Z_{y 2 \psi}=Z_{2 \psi_{R}}=Z_{2 \psi_{L}}=\mathcal{O}\left(\delta^{-1}\right)$.

Gauge boson self energies: $\boldsymbol{\Pi}_{\boldsymbol{A}}$. As noted before, in all loops containing gauge-Higgs interactions we can use a $\mathrm{U}(1)$ model and sum over the three gauge couplings. This takes care of the first three lines in eq. (A.18). To generalize the contribution from the fermion line we have to add the appropiate group factors. Finally, there are also new diagrams with gauge loops due to the non-abelian gauge interactions that can contribute to the wave-function normalization. Using the parameteric arguments in the paragraph below eq. (4.13), it is easy to see that these contributions are subdominant. Hence, just as for the fermion self-energy we conclude that for our set-up the SM generalization may change the coefficients and add new terms to the self-energy, but all terms can be neglected at leading order.

With the couplings given in A.3, the self-energies in the large field limit in the full SM are for case B:

$$
\begin{aligned}
\Pi_{Q^{\phi}}^{\mathrm{SM}} & =p^{2} \delta\left[-\left(Z_{2 Q^{\phi}}-1\right)+\mathcal{O}\left(\delta^{-1}\right)\right]+\phi^{2}\left[-\left(Z_{\lambda 2 Q^{\phi}}-1\right) \lambda+\frac{1}{8 \pi^{2} \epsilon}\left(\sum_{i} \frac{3 g_{i}^{4}}{\alpha_{0}}-N_{c} y^{4}\right)\right] \\
\Pi_{Q^{\theta}}^{\mathrm{SM}} & =p^{2} \delta\left[-\left(Z_{2 Q^{\theta}}-1\right)+\mathcal{O}\left(\delta^{-1}\right)\right]+3 \phi^{2}\left[-\left(Z_{\lambda 2 Q^{\theta}}-1\right) \lambda+\frac{1}{8 \pi^{2} \epsilon}\left(\sum_{i} \frac{3 g_{i}^{4}}{\alpha_{0}}-N_{c} y^{4}\right)\right] \\
\Pi_{\psi}^{\mathrm{SM}} & =\not p\left[-P_{L}\left(Z_{2 \psi_{L}}-1\right)-P_{R}\left(Z_{2 \psi_{R}}-1\right)+\mathcal{O}\left(\delta^{-1}\right)\right]+\phi\left[-\left(Z_{m 2 \psi}-1\right) \frac{y}{\sqrt{2}}+\mathcal{O}\left(\delta^{-1}\right)\right] \\
\Pi_{\mu \nu}^{A, \mathrm{SM}} & =\left(p^{2} g_{\mu \nu}-p_{\mu} p_{\nu}\right) \delta \alpha_{0}\left[-\left(Z_{2 Q^{A}}-1\right)+\mathcal{O}\left(\delta^{-1}\right)\right]+g_{\mu \nu} \phi^{2} \delta\left[-\left(Z_{m_{A}^{2}}-1\right) g^{2}+\mathcal{O}\left(\delta^{-1}\right)\right] .
\end{aligned}
$$


Case A can be obainted by setting $g \rightarrow 0$, case $\mathrm{C}$ by setting $y \rightarrow 0$, and case $\mathrm{D}$ by setting $y, g \rightarrow 0$.

Open Access. This article is distributed under the terms of the Creative Commons Attribution License (CC-BY 4.0), which permits any use, distribution and reproduction in any medium, provided the original author(s) and source are credited.

\section{References}

[1] PlancK collaboration, Planck 2018 results. X. Constraints on inflation, arXiv:1807.06211 [INSPIRE].

[2] J. Martin, C. Ringeval and V. Vennin, Encyclopadia Inflationaris, Phys. Dark Univ. 5-6 (2014) 75 [arXiv: 1303.3787] [INSPIRE].

[3] E.J. Copeland, A.R. Liddle, D.H. Lyth, E.D. Stewart and D. Wands, False vacuum inflation with Einstein gravity, Phys. Rev. D 49 (1994) 6410 [astro-ph/9401011] [INSPIRE].

[4] D. Baumann and L. McAllister, Inflation and String Theory, in Cambridge Monographs on Mathematical Physics, Cambridge University Press (2015) [arXiv:1404.2601] [INSPIRE].

[5] C.P. Burgess, S.P. Patil and M. Trott, On the Predictiveness of Single-Field Inflationary Models, JHEP 06 (2014) 010 [arXiv:1402.1476] [INSPIRE].

[6] J. Fumagalli and M. Postma, UV (in)sensitivity of Higgs inflation, JHEP 05 (2016) 049 [arXiv: 1602.07234] [INSPIRE].

[7] C. Germani and A. Kehagias, New Model of Inflation with Non-minimal Derivative Coupling of Standard Model Higgs Boson to Gravity, Phys. Rev. Lett. 105 (2010) 011302 [arXiv: 1003.2635] [INSPIRE].

[8] C. Germani, Spontaneous localization on a brane via a gravitational mechanism, Phys. Rev. D 85 (2012) 055025 [arXiv:1109.3718] [INSPIRE].

[9] S. Di Vita and C. Germani, Electroweak vacuum stability and inflation via nonminimal derivative couplings to gravity, Phys. Rev. D 93 (2016) 045005 [arXiv:1508.04777] [INSPIRE].

[10] E.E. Jenkins, A.V. Manohar and M. Trott, Renormalization Group Evolution of the Standard Model Dimension Six Operators I: Formalism and lambda Dependence, JHEP 10 (2013) 087 [arXiv: 1308.2627] [INSPIRE].

[11] F. Bezrukov, J. Rubio and M. Shaposhnikov, Living beyond the edge: Higgs inflation and vacuum metastability, Phys. Rev. D 92 (2015) 083512 [arXiv:1412.3811] [InSPIRE].

[12] F. Bezrukov and M. Shaposhnikov, Higgs inflation at the critical point, Phys. Lett. B 734 (2014) 249 [arXiv: 1403.6078] [INSPIRE].

[13] V.-M. Enckell, K. Enqvist and S. Nurmi, Observational signatures of Higgs inflation, JCAP 07 (2016) 047 [arXiv: 1603.07572] [INSPIRE].

[14] F. Bezrukov, M. Pauly and J. Rubio, On the robustness of the primordial power spectrum in renormalized Higgs inflation, JCAP 02 (2018) 040 [arXiv:1706. 05007] [INSPIRE].

[15] J. Fumagalli, S. Mooij and M. Postma, Unitarity and predictiveness in new Higgs inflation, JHEP 03 (2018) 038 [arXiv:1711.08761] [INSPIRE]. 
[16] F.L. Bezrukov and M. Shaposhnikov, The Standard Model Higgs boson as the inflaton, Phys. Lett. B 659 (2008) 703 [arXiv:0710.3755] [INSPIRE].

[17] K. Kamada, T. Kobayashi, T. Takahashi, M. Yamaguchi and J. Yokoyama, Generalized Higgs inflation, Phys. Rev. D 86 (2012) 023504 [arXiv:1203.4059] [INSPIRE].

[18] J. Rubio, Higgs inflation, Front. Astron. Space Sci. 5 (2019) 50 [arXiv:1807.02376] [INSPIRE].

[19] F. Bezrukov and M. Shaposhnikov, Standard Model Higgs boson mass from inflation: Two loop analysis, JHEP 07 (2009) 089 [arXiv:0904.1537] [inSPIRE].

[20] F. Bezrukov, A. Magnin, M. Shaposhnikov and S. Sibiryakov, Higgs inflation: consistency and generalisations, JHEP 01 (2011) 016 [arXiv: 1008.5157] [INSPIRE].

[21] A. De Simone, M.P. Hertzberg and F. Wilczek, Running Inflation in the Standard Model, Phys. Lett. B 678 (2009) 1 [arXiv:0812.4946] [INSPIRE].

[22] A.O. Bärvinsky, A.Y. Kamenshchik and A.A. Starobinsky, Inflation scenario via the Standard Model Higgs boson and LHC, JCAP 11 (2008) 021 [arXiv:0809.2104] [INSPIRE].

[23] A.O. Bärvinsky, A.Y. Kamenshchik, C. Kiefer, A.A. Starobinsky and C. Steinwachs, Asymptotic freedom in inflationary cosmology with a non-minimally coupled Higgs field, JCAP 12 (2009) 003 [arXiv:0904.1698] [inSPIRE].

[24] A.O. Bärvinsky, A.Y. Kamenshchik, C. Kiefer, A.A. Starobinsky and C.F. Steinwachs, Higgs boson, renormalization group, and naturalness in cosmology, Eur. Phys. J. C $\mathbf{7 2}$ (2012) 2219 [arXiv:0910.1041] [INSPIRE].

[25] D.P. George, S. Mooij and M. Postma, Quantum corrections in Higgs inflation: the real scalar case, JCAP 02 (2014) 024 [arXiv:1310.2157] [INSPIRE].

[26] D.P. George, S. Mooij and M. Postma, Quantum corrections in Higgs inflation: the Standard Model case, JCAP 04 (2016) 006 [arXiv: 1508.04660] [INSPIRE].

[27] M.P. Hertzberg, Can Inflation be Connected to Low Energy Particle Physics?, JCAP 08 (2012) 008 [arXiv:1110.5650] [inSPIRE].

[28] J.L.F. Barbón, J.A. Casas, J. Elias-Miro and J.R. Espinosa, Higgs Inflation as a Mirage, JHEP 09 (2015) 027 [arXiv: 1501.02231] [INSPIRE].

[29] J. Fumagalli, Renormalization Group independence of Cosmological Attractors, Phys. Lett. B 769 (2017) 451 [arXiv: 1611.04997] [INSPIRE].

[30] F. Bauer and D.A. Demir, Inflation with Non-Minimal Coupling: Metric versus Palatini Formulations, Phys. Lett. B 665 (2008) 222 [arXiv:0803.2664] [INSPIRE].

[31] S. Räsänen and P. Wahlman, Higgs inflation with loop corrections in the Palatini formulation, JCAP 11 (2017) 047 [arXiv: 1709.07853] [INSPIRE].

[32] V.-M. Enckell, K. Enqvist, S. Räsänen and E. Tomberg, Higgs inflation at the hilltop, JCAP 06 (2018) 005 [arXiv: 1802.09299] [INSPIRE].

[33] S. Räsänen, Higgs inflation in the Palatini formulation with kinetic terms for the metric, Open J. Astrophys. 2 (2019) 1 [arXiv:1811.09514] [INSPIRE].

[34] A. Racioppi, Non-Minimal (Self-)Running Inflation: Metric vs. Palatini Formulation, arXiv: 1912.10038 [INSPIRE]. 
[35] F. Bauer and D.A. Demir, Higgs-Palatini Inflation and Unitarity, Phys. Lett. B 698 (2011) 425 [arXiv: 1012.2900] [INSPIRE].

[36] A. Escrivà and C. Germani, Beyond dimensional analysis: Higgs and new Higgs inflations do not violate unitarity, Phys. Rev. D 95 (2017) 123526 [arXiv:1612.06253] [INSPIRE].

[37] M. Galante, R. Kallosh, A. Linde and D. Roest, Unity of Cosmological Inflation Attractors, Phys. Rev. Lett. 114 (2015) 141302 [arXiv:1412.3797] [INSPIRE].

[38] G.A. Vilkovisky, The Unique Effective Action in Quantum Field Theory, Nucl. Phys. B 234 (1984) 125 [INSPIRE].

[39] J.-O. Gong and T. Tanaka, A covariant approach to general field space metric in multi-field inflation, JCAP 03 (2011) 015 [Erratum JCAP 02 (2012) E01] [arXiv:1101.4809] [INSPIRE].

[40] J. Fumagalli, S. Renaux-Petel and J.W. Ronayne, Higgs vacuum (in)stability during inflation: the dangerous relevance of de Sitter departure and Planck-suppressed operators, JHEP 02 (2020) 142 [arXiv: 1910.13430] [INSPIRE].

[41] B.S. DeWitt, Dynamical theory of groups and fields, Conf. Proc. C 630701 (1964) 585 [INSPIRE].

[42] M. Sasaki and E.D. Stewart, A General analytic formula for the spectral index of the density perturbations produced during inflation, Prog. Theor. Phys. 95 (1996) 71 [astro-ph/9507001] [INSPIRE].

[43] S. Groot Nibbelink and B.J.W. van Tent, Scalar perturbations during multiple field slow-roll inflation, Class. Quant. Grav. 19 (2002) 613 [hep-ph/0107272] [INSPIRE].

[44] A. Achucarro, J.-O. Gong, S. Hardeman, G.A. Palma and S.P. Patil, Features of heavy physics in the CMB power spectrum, JCAP 01 (2011) 030 [arXiv:1010.3693] [INSPIRE].

[45] R. Alonso, E.E. Jenkins and A.V. Manohar, A Geometric Formulation of Higgs Effective Field Theory: Measuring the Curvature of Scalar Field Space, Phys. Lett. B 754 (2016) 335 [arXiv: 1511.00724] [INSPIRE].

[46] R. Alonso, E.E. Jenkins and A.V. Manohar, Geometry of the Scalar Sector, JHEP 08 (2016) 101 [arXiv: 1605. 03602] [INSPIRE].

[47] A. Helset, M. Paraskevas and M. Trott, Gauge fixing the Standard Model Effective Field Theory, Phys. Rev. Lett. 120 (2018) 251801 [arXiv: 1803.08001] [INSPIRE].

[48] R. Nagai, M. Tanabashi, K. Tsumura and Y. Uchida, Symmetry and geometry in a generalized Higgs effective field theory: Finiteness of oblique corrections versus perturbative unitarity, Phys. Rev. D 100 (2019) 075020 [arXiv: 1904.07618] [INSPIRE].

[49] A. Helset, A. Martin and M. Trott, The Geometric Standard Model Effective Field Theory, JHEP 03 (2020) 163 [arXiv:2001.01453] [INSPIRE].

[50] R. Alonso, A covariant momentum representation for loop corrections in gravity, JHEP 05 (2020) 131 [arXiv: 1912.09671] [INSPIRE].

[51] C.F. Steinwachs and A.Y. Kamenshchik, Non-minimal Higgs Inflation and Frame Dependence in Cosmology, AIP Conf. Proc. 1514 (2013) 161 [arXiv:1301.5543] [InSPIRE].

[52] K. Falls and M. Herrero-Valea, Frame (In)equivalence in Quantum Field Theory and Cosmology, Eur. Phys. J. C 79 (2019) 595 [arXiv:1812.08187] [InSPIRE]. 
[53] K. Finn, S. Karamitsos and A. Pilaftsis, Frame Covariance in Quantum Gravity, Phys. Rev. D 102 (2020) 045014 [arXiv: 1910.06661] [INSPIRE].

[54] E.S. Fradkin and A.A. Tseytlin, On the New Definition of Off-shell Effective Action, Nucl. Phys. B 234 (1984) 509 [INSPIRE].

[55] B.S. DeWitt, Quantum Theory of Gravity. 2. The Manifestly Covariant Theory, Phys. Rev. 162 (1967) 1195 [INSPIRE].

[56] D. Buttazzo et al., Investigating the near-criticality of the Higgs boson, JHEP 12 (2013) 089 [arXiv: 1307.3536] [INSPIRE].

[57] A.H. Hoang, The Top Mass: Interpretation and Theoretical Uncertainties, in proceedings of the 7th International Workshop on Top Quark Physics (TOP2014), Cannes, France, 28 September-3 October 2014, arXiv:1412.3649 [INSPIRE].

[58] P. Nason, The Top Mass in Hadronic Collisions, in From My Vast Repertoire... : Guido Altarelli's Legacy, A. Levy, S. Forte and G. Ridolfi eds., World Scientific (2019), pp. 123-151 [arXiv: 1712.02796] [INSPIRE].

[59] Particle Data Group, Review of Particle Physics, Phys. Rev. D 98 (2018) 030001 [INSPIRE].

[60] M.-x. Luo and Y. Xiao, Two loop renormalization group equations in the standard model, Phys. Rev. Lett. 90 (2003) 011601 [hep-ph/0207271] [INSPIRE].

[61] A. Urbano, Inflation without gauge redundancy, JCAP 04 (2020) 040 [arXiv: 2001.05480] [INSPIRE].

[62] Planck collaboration, Planck 2015 results. XX. Constraints on inflation, Astron. Astrophys. 594 (2016) A20 [arXiv: 1502.02114] [InSPIRE].

[63] M. Shaposhnikov, A. Shkerin and S. Zell, Quantum Effects in Palatini Higgs Inflation, JCAP 07 (2020) 064 [arXiv: 2002.07105] [INSPIRE]. 